

\title{
The Picard rank conjecture for the Hurwitz spaces of degree up to five
}

\author{
Anand Deopurkar and Anand Patel
}

We prove that the rational Picard group of the simple Hurwitz space $\mathcal{H}_{d, g}$ is trivial for $d$ up to five. We also relate the rational Picard groups of the Hurwitz spaces to the rational Picard groups of the Severi varieties of nodal curves on Hirzebruch surfaces.

\section{Introduction}

Let $\mathcal{H}_{d, g}$ be the simple Hurwitz space which parametrizes isomorphism classes of simply branched degree- $d$ covers of genus-zero curves by genus- $g$ curves. Although $\mathcal{H}_{d, g}$ has been studied classically, many fundamental questions about its geometry are still unanswered. The goal of this paper is to address one such question: the question of its Picard group. It is conjectured (for example, [Diaz and Edidin 1996]) that the rational Picard group $\operatorname{Pic}_{\mathbb{Q}}\left(\mathcal{H}_{d, g}\right)$ is trivial. We call this the Picard rank conjecture for $\mathcal{H}_{d, g}$. Our main result is a proof of this conjecture for $d \leq 5$.

Theorem A. The rational Picard group of $\mathcal{H}_{d, g}$ is trivial for $d \leq 5$.

In the main text, Theorem A is divided into the case of degree 3 (Proposition 3.3), degree 4 (Proposition 4.10), and degree 5 (Proposition 5.4).

The Picard rank conjecture was known for $d=2$ and 3. For $d=2$, it was proved by Cornalba and Harris [1988, Lemma 4.5], and for $d=3$ by Stankova-Frenkel [Stankova-Frenkel 2000, §12.2]. In these cases, there are now more refined results about the moduli stacks; see [Cornalba 2007] for $d=2$ and [Bolognesi and Vistoli 2012; Bolognesi and Lönne 2014] for $d=3$.

The conjecture is also known for $d>2 g-2$. In this range, the map $\mathcal{H}_{d, g} \rightarrow \mathcal{M}_{g}$ is a fibration, where $\mathcal{M}_{g}$ is the moduli space of smooth curves of genus $g$. An analysis of this fibration shows that $\operatorname{Pic}_{\mathbb{Q}}\left(\mathcal{H}_{d, g}\right)=0$ if and only if $\operatorname{Pic}_{\mathbb{Q}}\left(\mathcal{M}_{g}\right) \cong \mathbb{Q}$ (see, for example, [Mochizuki 1995] or [Diaz and Edidin 1996, §3]). Thus, the conjecture for $d>2 g-2$ follows from Harer's theorem [1983]. 
We briefly explain the rationale behind the conjecture. Let us blur the distinction between the coarse moduli spaces and the fine moduli stacks. This is harmless, since we are concerned with the rational Picard group. Let us also take $d \geq 4$ (the discussion holds for $d=2,3$ with minor modifications). Denote by $\tilde{\mathcal{H}}_{d, g}$ the partial compactification of $\mathcal{H}_{d, g}$ that parametrizes covers $\left[\alpha: C \rightarrow \mathbb{P}^{1}\right]$ where $C$ is allowed to be nodal, but still irreducible, and $\alpha$ need not be simply branched. Let $\alpha: \mathcal{C} \rightarrow \mathcal{P}$ be the universal family over $\widetilde{\mathcal{H}}_{d, g}$, where $\rho: \mathcal{C} \rightarrow \widetilde{\mathcal{H}}_{d, g}$ is a family of irreducible, at worst nodal curves of arithmetic genus $g$, and $\pi: \mathcal{P} \rightarrow \widetilde{\mathcal{H}}_{d, g}$ a family of smooth curves of genus 0 . From this data, we can construct three "tautological" divisor classes on $\widetilde{\mathcal{H}}_{d, g}$, given by

$$
\rho_{*}\left(c_{1}\left(\omega_{\rho}\right)^{2}\right), \quad \rho_{*}\left(c_{1}\left(\omega_{\rho}\right) \alpha^{*} c_{1}\left(\omega_{\pi}\right)\right), \quad \text { and } \quad \rho_{*}\left(\left[\delta_{\rho}\right]\right) .
$$

Here $\omega$ stands for the relative dualizing sheaf and $\delta$ for the singular locus. It is easy to check that the three tautological classes are $\mathbb{Q}$-linearly independent. On the other hand, $\tilde{\mathcal{H}}_{d, g} \backslash \mathcal{H}_{d, g}$ is a union of three irreducible divisors, namely, the locus $\Delta$ where $C$ is singular, the locus $T$ where $\alpha$ has a higher order ramification point, and the locus $D$ where $\alpha$ has two ramification points over a branch point. It is also easy to check that the classes of $\Delta, T$, and $D$ are $\mathbb{Q}$-linearly independent. Thus, $\operatorname{Pic}_{\mathbb{Q}}\left(\mathcal{H}_{d, g}\right)=0$ is equivalent to $\operatorname{Pic}_{\mathbb{Q}}\left(\widetilde{\mathcal{H}}_{d, g}\right)$ being generated by the tautological classes. The Picard rank conjecture thus expresses the often-satisfied expectation that there are no other divisor classes than the tautological ones.

We now outline our strategy for proving Theorem A. Let $\alpha: C \rightarrow \mathbb{P}^{1}$ be a degree$d$ cover. Then $C$ embeds in a $\mathbb{P}^{d-2}$-bundle over $\mathbb{P}^{1}$, which we denote by $\mathbb{P} E \rightarrow \mathbb{P}^{1}$. Thanks to the work of Casnati and Ekedahl, the resolution of the ideal of $C$ in $\mathbb{P} E$ can be described explicitly. The terms in this resolution involve (twists of) vector bundles on $\mathbb{P}^{1}$ [Casnati and Ekedahl 1996]. Let $U \subset \tilde{\mathcal{H}}_{d, g}$ be the open locus where these vector bundles are the most generic. The key steps in our proof are the following:

(1) Identify the divisorial components of $\tilde{\mathcal{H}}_{d, g} \backslash U$.

(2) Express $U$ as a (successive) quotient of an open subset of an affine space by actions of linear algebraic groups.

(3) Use the previous two steps to get a bound on the Picard rank of $\widetilde{\mathcal{H}}_{d, g}$, and in turn, the Picard rank of $\mathcal{H}_{d, g}$.

Needless to say, we are able to carry out all three steps only for $d \leq 5$. However, we can carry out parts of step (1) in general. For step (2), we highlight that it remains unknown in general whether one can dominate $\widetilde{\mathcal{H}}_{d, g}$ by an affine space for $d \geq 6$.

To analyze $\widetilde{\mathcal{H}}_{d, g} \backslash U$, we must analyze the loci in $\widetilde{\mathcal{H}}_{d, g}$ where the bundle $E$ and the vector bundles appearing in the resolution of $C$ are unbalanced. We call these loci the Maroni loci and the Casnati-Ekedahl loci, respectively. We spend significant 
effort on understanding the decomposition of $\tilde{\mathcal{H}}_{d, g}$ into these loci. Contained in Section 2, the results of this analysis may be of independent interest.

A key tool in our analysis is a construction that relates the Maroni loci to the Severi varieties of Hirzebruch surfaces. Originally due to Ohbuchi [1997], this "associated scroll construction" allows us to get the required dimension estimates. The key input here is a theorem of Tyomkin [2007] that guarantees that the Severi varieties of Hirzebruch surfaces are irreducible of the expected dimension.

The associated scroll construction also lets us relate the Picard ranks of the Hurwitz spaces to the Picard ranks of the Severi varieties. To state our result, let us denote by $\mathcal{U}_{g}\left(\mathbb{F}_{m}, d \tau\right)$ the space of irreducible nodal curves of geometric genus $g$ in the linear system $|d \tau|$ on the Hirzebruch surface $\mathbb{F}_{m}$, where $\tau$ is the section with self-intersection $m$.

Theorem B. Let $m \geq\lfloor(g+d-1) /(d-1)\rfloor$. Then $\operatorname{Pic}_{\mathbb{Q}} \mathcal{U}_{g}\left(\mathbb{F}_{m}, d \tau\right)=0$ implies $\operatorname{Pic}_{\mathbb{Q}} \mathcal{H}_{d, g}=0$.

Let $m \geq\lceil 2(g+d-1) /(d-1)\rceil$. Then $\operatorname{Pic}_{\mathbb{Q}} \mathcal{U}_{g}\left(\mathbb{F}_{m}, d \tau\right)=0$ if and only if $\operatorname{Pic}_{\mathbb{Q}} \mathcal{H}_{d, g}=0$.

In the main text, Theorem B is Theorem 6.7.

Notation. We work with a few different versions of the Hurwitz spaces. We assemble their definitions here. We work over the field $\mathbb{C}$ of complex numbers. By a curve, we mean a connected, proper, reduced scheme of finite type over $\mathbb{C}$. Throughout, assume that $g \geq 3$.

$\mathcal{H}_{d, g}$ : This is the coarse moduli space of $\left[\alpha: C \rightarrow \mathbb{P}^{1}\right]$, where $C$ is a smooth curve of genus $g$ and $\alpha$ a finite map of degree $d$ with simple branching (that is, the branch divisor of $\alpha$ is supported at $2 g+2 d-2$ distinct points). Two such covers $\left[\alpha_{1}: C_{1} \rightarrow \mathbb{P}^{1}\right]$ and $\left[\alpha_{2}: C_{2} \rightarrow \mathbb{P}^{1}\right]$ are considered isomorphic if there are isomorphisms $\phi: C_{1} \rightarrow C_{2}$ and $\psi: \mathbb{P}^{1} \rightarrow \mathbb{P}^{1}$ such that $\alpha_{2} \circ \phi=\psi \circ \alpha_{1}$.

$\tilde{\mathcal{H}}_{d, g}$ : This is the coarse moduli space of $\left[\alpha: C \rightarrow \mathbb{P}^{1}\right]$, where $C$ is an irreducible, at worst nodal curve of arithmetic genus $g$, and $\alpha$ a finite map of degree $d$. The isomorphism condition is the same as that for $\mathcal{H}_{d, g}$.

$\mathcal{H}_{d, g}^{\dagger}$ : This is like $\mathcal{H}_{d, g}$, but with "framed" target $\mathbb{P}^{1}$. The objects it parametrizes are $\left[\alpha: C \rightarrow \mathbb{P}^{1}\right]$ as in the description of $\mathcal{H}_{d, g}$, but $\left[\alpha_{1}: C_{1} \rightarrow \mathbb{P}^{1}\right]$ and $\left[\alpha_{2}: C_{2} \rightarrow \mathbb{P}^{1}\right]$ are considered isomorphic if there is an isomorphism $\phi:$ $C_{1} \rightarrow C_{2}$ such that $\alpha_{2} \circ \phi=\alpha_{1}$.

$\tilde{\mathcal{H}}_{d, g}^{\dagger}$ : This is like $\tilde{\mathcal{H}}_{d, g}$, but with framed target $\mathbb{P}^{1}$.

All four are irreducible quasiprojective varieties with at worst quotient singularities. In particular, they are normal and $\mathbb{Q}$-factorial. The group Aut $\mathbb{P}^{1}=\mathrm{PGL}_{2}$ acts on the framed versions. The unframed versions are the quotients by this action in 
the sense that the fibers of the morphism from the framed space to the unframed space are precisely the $\mathrm{PGL}_{2}$ orbits. We have

$$
\operatorname{dim} \mathcal{H}_{d, g}=\operatorname{dim} \tilde{\mathcal{H}}_{d, g}=2 g+2 d-5
$$

and

$$
\operatorname{dim} \mathcal{H}_{d, g}^{\dagger}=\operatorname{dim} \tilde{\mathcal{H}}_{d, g}^{\dagger}=2 g+2 d-2
$$

In addition, we work with the following Severi varieties:

$\mathcal{U}_{g}\left(\mathbb{F}_{m}, d \tau\right)$ : This is the locus of irreducible nodal curves of geometric genus $g$ in the linear series $|d \tau|$ in the Hirzebruch surface $\mathbb{F}_{m}$. Here $\tau \subset \mathbb{F}_{m}$ is the section of self-intersection $m$.

$\mathcal{V}_{g}\left(\mathbb{F}_{m}, d \tau\right)$ : This is the closure of $\mathcal{U}_{g}\left(\mathbb{F}_{m}, d \tau\right)$ in the projective space $|d \tau|$.

$\mathcal{V}_{g}^{\text {irr }}\left(\mathbb{F}_{m}, d \tau\right)$ : This is the open subset of reduced irreducible curves in $\mathcal{V}_{g}\left(\mathbb{F}_{m}, d \tau\right)$.

We do not distinguish between a vector bundle and the corresponding locally free sheaf. Note that the vector bundle associated to the locally free sheaf $F$ is the relative Spec of the symmetric algebra on $F^{\vee}$.

\section{Preliminaries}

In this expository section, we recall two key results. The first describes the Picard group of the quotient of a variety by a group action. The second is a structure theorem for finite covers which enables us to describe a large open subset of the Hurwitz space as such a quotient.

1A. Picard groups of quotients. Let $G$ be a linear algebraic group acting on a variety $X$. Denote by $\operatorname{Pic}_{G} X$ the group of $G$-linearized line bundles on $X$. Forgetting the $G$-linearization gives a homomorphism $\operatorname{Pic}_{G} X \rightarrow \operatorname{Pic} X$.

Proposition 1.1 [Knop et al. 1989, Lemma 2.2, Proposition 2.3]. For a connected linear algebraic group $G$ acting on an irreducible variety $X$, we have an exact sequence

$$
\chi(G) \longrightarrow \operatorname{Pic}_{G} X \longrightarrow \operatorname{Pic} X,
$$

where $\chi(G)$ is the group of (algebraic) characters of $G$. Furthermore, if $X$ is normal, then the sequence has an extension by a homomorphism $\operatorname{Pic} X \rightarrow \operatorname{Pic} G$.

Let $\pi: X \rightarrow Y$ be a morphism that is equivariant with the trivial $G$ action on $Y$. Let $L$ be a line bundle on $Y$. The pullback $\pi^{*} L$ carries a natural $G$-linearization. We thus have a homomorphism Pic $Y \rightarrow \operatorname{Pic}_{G} X$. 
Proposition 1.2. Let $X$ and $Y$ be irreducible normal varieties, $G$ a linear algebraic group acting on $X$, and $\pi: X \rightarrow Y$ a surjective morphism, equivariant with the trivial action on $Y$. Suppose the fibers of $\pi$ consist of single $G$-orbits. Then the map Pic $Y \rightarrow \operatorname{Pic}_{G} X$ is injective and we have

$$
\text { rk Pic } Y \leq \operatorname{rk} \chi(G)+\operatorname{rk} \operatorname{Pic} X \text {. }
$$

Furthermore, if $G$ is reductive and the stabilizers $G_{x}$ are finite, then we have an isomorphism

$$
\operatorname{Pic} Y \otimes \mathbb{Q} \stackrel{\sim}{\longrightarrow} \operatorname{Pic}_{G} X \otimes \mathbb{Q} \text {. }
$$

Proof. Suppose $L$ is a line bundle on $Y$ such that $\pi^{*} L$ is trivial as a $G$-linearized line bundle. Then $\pi^{*} L$ has a $G$-invariant nowhere-vanishing section. We claim that such a section descends to a nowhere-vanishing section of $L$ on $Y$. The crucial point is that in our setup, $Y$ is a geometric quotient of $X$ [Mumford et al. 1994, Proposition 0.2]. That is, for every open $U \subset Y$, the preimage $\pi^{-1} U$ is open and the functions on $U$ are the invariant functions on $\pi^{-1} U$ :

$$
\Gamma\left(U, \mathcal{O}_{Y}\right)=\Gamma\left(\pi^{-1} U, \mathcal{O}_{X}\right)^{G} .
$$

It follows that the sections of $L$ on $U$ are the invariant sections of $\pi^{*} L$ on $\pi^{-1}(U)$ :

$$
\Gamma(U, L)=\Gamma\left(\pi^{-1} U, \pi^{*} L\right)^{G} .
$$

Thus, a $G$-invariant section $\sigma$ of $\pi^{*} L$ on $X$ gives a section $\bar{\sigma}$ of $L$ on $Y$. It is easy to check that if $\sigma$ is nowhere-vanishing, so is $\bar{\sigma}$.

The bound on rk Pic $Y$ follows from injectivity and Proposition 1.1. For the last statement, we use the characterization of the image of Pic $Y \rightarrow \operatorname{Pic}_{G} X$ from [Knop et al. 1989, Proposition 4.2]: a $G$-linearized line bundle $L$ is in the image if and only if for every $x \in X$, the stabilizer group $G_{x}$ acts trivially on the fiber $L_{x}$. Since the stabilizers are finite, we can arrange this by passing to a large enough power of $L$.

We end with a simple application:

Proposition 1.3. Let $U \subset \tilde{\mathcal{H}}_{d, g}$ be any open subset and $U^{\dagger}$ its preimage under $\tilde{\mathcal{H}}_{d, g}^{\dagger} \rightarrow \widetilde{\mathcal{H}}_{d, g}$. Then

$$
\operatorname{rk} \operatorname{Pic} U=\operatorname{rkPic} U^{\dagger} \text {. }
$$

Proof. Apply Propositions 1.1 and 1.2 with $G=\mathrm{PGL}_{2}, X=U^{\dagger}$, and $Y=U$.

1B. The Casnati-Ekedahl structure theorem. Let $X$ and $Y$ be integral schemes and $\alpha: X \rightarrow Y$ a finite flat Gorenstein morphism of degree $d \geq 3$. The map $\alpha$ gives an exact sequence

$$
0 \longrightarrow \mathcal{O}_{Y} \longrightarrow \alpha_{*} \mathcal{O}_{X} \longrightarrow E_{\alpha}^{\vee} \longrightarrow 0
$$


where $E=E_{\alpha}$ is a vector bundle of rank $d-1$ on $Y$, called the Tschirnhausen bundle of $\alpha$. Denote by $\omega_{\alpha}$ the dualizing sheaf of $\alpha$. Applying $\operatorname{Hom}_{Y}\left(-, \mathcal{O}_{Y}\right)$ to (1-1), we get

$$
0 \longrightarrow E \longrightarrow \alpha_{*} \omega_{\alpha} \longrightarrow \mathcal{O}_{Y} \longrightarrow 0 .
$$

The map $E \rightarrow \alpha_{*} \omega_{\alpha}$ induces a map $\alpha^{*} E \rightarrow \omega_{\alpha}$.

Theorem 1.4 [Casnati and Ekedahl 1996, Theorem 2.1]. In the above setup, $\alpha^{*} E \rightarrow$ $\omega_{\alpha}$ gives an embedding $\iota: X \rightarrow \mathbb{P} E$ with $\alpha=\pi \circ \iota$, where $\pi: \mathbb{P} E \rightarrow Y$ is the projection. Moreover, the subscheme $X \subset \mathbb{P} E$ can be described as follows:

(1) The resolution of $\mathcal{O}_{X}$ as an $\mathcal{O}_{\mathbb{P} E}$-module has the form

$$
\begin{aligned}
& 0 \longrightarrow \pi^{*} N_{d-2}(-d) \longrightarrow \pi^{*} N_{d-3}(-d+2) \longrightarrow \pi^{*} N_{d-4}(-d+3) \\
& \longrightarrow \cdots \longrightarrow \pi^{*} N_{2}(-3) \longrightarrow \pi^{*} N_{1}(-2) \longrightarrow \mathcal{O}_{\mathbb{P} E} \longrightarrow \mathcal{O}_{X} \longrightarrow 0,
\end{aligned}
$$

where the $N_{i}$ are vector bundles on $Y$. Restricted to a point $y \in Y$, this sequence is the minimal free resolution of $X_{y} \subset \mathbb{P} E_{y}$.

(2) The ranks of the $N_{i}$ are given by

$$
\operatorname{rk} N_{i}=\frac{i(d-2-i)}{d-1}\left(\begin{array}{c}
d \\
i+1
\end{array}\right)
$$

(3) We have $N_{d-2} \cong \pi^{*} \operatorname{det} E$. Furthermore, the resolution is symmetric, that is, isomorphic to the resolution obtained by applying $\operatorname{Hom}_{\mathcal{O}_{\mathbb{P} E}}\left(-, N_{d-2}(-d)\right)$.

The branch divisor of $\alpha: X \rightarrow Y$ is given by a section of $(\operatorname{det} E)^{\otimes 2}$. In particular, if $X$ is a curve of (arithmetic) genus $g, \alpha$ has degree $d$, and $Y=\mathbb{P}^{1}$, then

$$
\text { rk } E=d-1 \text { and } \operatorname{deg} E=g+d-1 \text {. }
$$

\section{The Maroni and Casnati-Ekedahl loci}

Consider a cover $\alpha: C \rightarrow \mathbb{P}^{1}$ and the relative canonical embedding $C \subset \mathbb{P} E_{\alpha}$. Since vector bundles on $\mathbb{P}^{1}$ split as direct sums of line bundles, the vector bundle $E_{\alpha}$, and the higher syzygy bundles $N_{i}$ appearing in Theorem 1.4, are discrete invariants of $\alpha$. We thus get a decomposition of the Hurwitz space into locally closed subsets where the isomorphism type of the bundles $E_{\alpha}$ and $N_{i}$ are constant. This section is devoted to the analysis of some of these locally closed subvarieties, particularly their dimensions. We only consider the bundle $E_{\alpha}$ and $F_{\alpha}:=N_{1}$. Note that

$$
E_{\alpha}=\operatorname{ker}\left(\alpha_{*} \omega_{\alpha} \rightarrow \mathcal{O}_{Y}\right) \text { and } F_{\alpha}=\alpha_{*} I_{C}(2),
$$

where $I_{C} \subset \mathcal{O}_{\mathbb{P} E_{\alpha}}$ is the ideal sheaf of $C$. 
Definition 2.1. For vector bundles $E$ and $F$ on $\mathbb{P}^{1}$, define closed subvarieties of $\mathcal{H}_{d, g}^{\dagger}$

$$
\begin{aligned}
M(E, F) & :=\overline{\left\{\left[\alpha: C \rightarrow \mathbb{P}^{1}\right] \mid E_{\alpha} \cong E \text { and } F_{\alpha} \cong F\right\}}, \\
M(E) & :=\overline{\left\{\left[\alpha: C \rightarrow \mathbb{P}^{1}\right] \mid E_{\alpha} \cong E\right\}}, \\
C(F) & :=\overline{\left\{\left[\alpha: C \rightarrow \mathbb{P}^{1}\right] \mid F_{\alpha} \cong F\right\}} .
\end{aligned}
$$

Call $M(E)$ the Maroni loci and $C(F)$ the Casnati-Ekedahl loci. Define subvarieties $\tilde{M}(E, F), \tilde{M}(E)$, and $\widetilde{C}(F)$ of $\widetilde{\mathcal{H}}_{d, g}^{\dagger}$ analogously.

Abusing notation, we denote the images of these loci in the unframed versions $\mathcal{H}_{d, g}$ and $\widetilde{\mathcal{H}}_{d, g}$ by the same letters. The framed versus unframed setting is usually clear by context, and sometimes irrelevant, for example in discussing the codimensions. We caution the reader that these loci are not necessarily irreducible or of expected dimension (Examples 4.3, 4.4). Even determining whether they are nonempty remains a challenge in full generality.

2A. The associated scroll construction. To analyze the Maroni loci $M(E)$, we associate to a cover of $\mathbb{P}^{1}$ a curve on a Hirzebruch surface. The construction is originally due to Ohbuchi [1997]. Let $C$ be an irreducible curve of arithmetic genus $g$ and $\alpha: C \rightarrow \mathbb{P}^{1}$ a finite cover of degree $d$. Let $\zeta$ be a global section of $\mathcal{O}_{C}(m)=\alpha^{*} \mathcal{O}_{\mathbb{P}^{1}}(m)$ that projects to a nonzero section of $E_{\alpha}^{\vee}(m)$. In other words, let $\zeta \in H^{0}\left(C, \mathcal{O}_{C}(m)\right)$ be an element not contained in $\alpha^{*} H^{0}\left(\mathbb{P}^{1}, \mathcal{O}_{\mathbb{P}^{1}}(m)\right)$. The section $\zeta$ gives a map from $C$ to the total space of the line bundle $\mathcal{O}(m)$ over $\mathbb{P}^{1}$. Let $\mathbb{F}_{m}=\operatorname{Proj}(\mathcal{O} \oplus \mathcal{O}(-m))$ be the Hirzebruch surface that compactifies this total space. We thus get the diagram

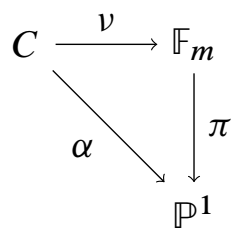

Let $\sigma \subset \mathbb{F}_{m}$ be the directrix (the unique section of $\mathbb{F}_{m} \rightarrow \mathbb{P}^{1}$ of negative selfintersection) and $\tau \subset \mathbb{F}_{m}$ the section disjoint from $\sigma$ (so that $\sigma^{2}=-m$ and $\tau^{2}=m$ ). By construction, $v(C) \subset \mathbb{F}_{m}$ avoids the directrix $\sigma$. Suppose $C$ is smooth and $\alpha: C \rightarrow \mathbb{P}^{1}$ does not factor nontrivially. Then $v$ is birational onto its image, and therefore $v(C)$ is a reduced and irreducible element of the linear system $|d \tau|$. By the following proposition, $v(C)$ is a point in the Severi variety $\mathcal{V}_{g}\left(\mathbb{F}_{m}, d \tau\right)$.

Proposition 2.2. A reduced and irreducible curve on $\mathbb{F}_{m}$ of geometric genus $g$ in the linear system $|d \tau|$ is a flat limit of irreducible nodal curves of geometric genus $g$. 
Proof. Let $\bar{C} \subset \mathbb{F}_{m}$ be such a reduced and irreducible curve. Let $C \rightarrow \bar{C}$ be the normalization map. Denote by $v$ the composite map $v: C \rightarrow \mathbb{F}_{m}$. Let $\mathcal{M}$ be a component of the Kontsevich space of maps $\mathcal{M}_{g}\left(\mathbb{F}_{m}, d \tau\right)$ containing $v$. Let $N_{v}$ be the normal sheaf of $v$; this is the cokernel of $T_{C} \rightarrow v^{*} T_{\mathbb{F}_{m}}$. Then, we have a lower bound $\operatorname{dim} \mathcal{M} \geq \chi\left(N_{v}\right)$. Since

$$
\chi\left(N_{v}\right)=\chi\left(v^{*} T_{\mathbb{F}_{m}}\right)-\chi\left(T_{C}\right)=g-\operatorname{deg}\left(K_{\mathbb{F}_{m}} \cdot \bar{C}\right)-1,
$$

we get

$$
\operatorname{dim} \mathcal{M} \geq g-\operatorname{deg}\left(K_{\mathbb{F}_{m}} \cdot \bar{C}\right)-1 .
$$

By [Harris 1986, Proposition 2.2], a general $v_{\text {gen }}: C_{\text {gen }} \rightarrow \mathbb{F}_{m}$ in $\mathcal{M}$ is birational onto its image and the image has only nodes as singularities.

We can make the construction in a family. Let $M$ be a reduced scheme, $\rho: C \rightarrow M$ a generically smooth family of reduced and irreducible curves of genus $g$, and $\alpha$ : $C \rightarrow \mathbb{P}^{1} \times M$ a finite flat $M$-morphism of degree $d$. Set $\mathcal{O}_{C}(m)=\alpha^{*} \mathcal{O}(m)$. Assume that none of the fibers $\alpha_{t}: C_{t} \rightarrow \mathbb{P}^{1}$ factor nontrivially and that $H^{0}\left(C_{t}, \mathcal{O}_{C_{t}}(m)\right)$ has constant rank. Then $\rho_{*} \mathcal{O}_{C}(m)$ is a vector bundle on $M$. The trivial subbundle $H^{0}\left(\mathbb{P}^{1}, \mathcal{O}(m)\right) \otimes \mathcal{O}_{M}$ maps injectively to $\rho_{*} \mathcal{O}_{C}(m)$. Let $U$ be the complement of the image of this map in the total space of $\rho_{*} \mathcal{O}_{C}(m)$. Fiberwise, the sections of $U$ correspond to the sections $\zeta$ which project nontrivially onto $E_{\alpha}^{\vee}(m)$. Then the associated scroll construction gives a morphism

$$
U \rightarrow \mathcal{V}_{g}\left(\mathbb{F}_{m}, d \tau\right) .
$$

We will use this construction where $M$ is a Maroni locus. As described, the construction depends on the existence of a universal family, and thus gives a morphism from the fine moduli stack. But since $\mathcal{V}_{g}\left(\mathbb{F}_{m}, d \tau\right)$ is a scheme, we get a canonical induced map from the coarse space.

The following crucial result makes the above construction useful:

Theorem 2.3 [Tyomkin 2007]. All Severi varieties parametrizing irreducible curves on Hirzebruch surfaces are irreducible and of expected dimension. In particular, the variety $\mathcal{V}_{g}\left(\mathbb{F}_{m}, d \tau\right)$ is irreducible of dimension $d m+2 d+g-1$.

We also need the following result, which we prove for lack of a reference:

Proposition 2.4. Let $\bar{C} \subset \mathbb{F}_{m}$ be a general point of $\mathcal{V}_{g}\left(\mathbb{F}_{m}, d \tau\right)$ and $C \rightarrow \bar{C}$ the normalization. Then the composite $C \rightarrow \mathbb{P}^{1}$ is simply branched.

Proof. In light of Theorem 2.3, it suffices to exhibit a particular $\bar{C}$ of geometric genus $g$ in $\mathcal{V}_{g}\left(\mathbb{F}_{m}, d \tau\right)$ whose normalization is simply branched over $\mathbb{P}^{1}$. One way is to start with $X=\mathbb{P}^{1}$ and $\alpha: X \rightarrow \mathbb{P}^{1}$ a simply branched cover of degree $d$. Then $E_{\alpha}=\mathcal{O}(1)^{\oplus(d-1)}$. Choosing a general section of $E_{\alpha}^{\vee}(m)$ gives $v: X \rightarrow \mathbb{F}_{m}$ such that $v(X)$ is nodal. It is easy to see that $v(X)$ is in the closure of $\mathcal{V}_{g}\left(\mathbb{F}_{m}, d \tau\right)$. Indeed, 
since the set of nodes of $v(X)$ impose independent conditions on $\left|K_{\mathbb{F}_{m}}+d \tau\right|$, they automatically impose independent conditions on $|d \tau|$ as well, and hence we may smooth out the required number of nodes of $v(X)$ to deform to a curve of geometric genus $g$. A general fiber of such a smoothing is the required $\bar{C}$.

Remark 2.5. We can realize the associated scroll construction geometrically as follows. The choice of a general global section $\zeta$ of $\mathcal{O}_{C}(m)$ can be thought of as a choice of a geometric section $\sigma: \mathbb{P}^{1} \rightarrow \mathbb{P} E$. In the $\mathbb{P}^{d-2}$ fibers of $\pi: \mathbb{P} E \rightarrow \mathbb{P}^{1}$, we now have $d+1$ points: $d$ points coming from the fibers of the map $\alpha: C \rightarrow \mathbb{P}^{1}$, and one more point provided by the section $\sigma$. For general $t \in \mathbb{P}^{1}$, these $d+1$ points will be in general position, and so will define a unique rational normal curve $R_{t} \subset \mathbb{P} E$. Consider the birationally ruled surface $S \subset \mathbb{P} E$ defined as the closure of the union of the $R_{t} . S$ contains both $\sigma$ and $C$, and is fibered over $\mathbb{P}^{1}$. We contract all components of the fibers of the projection $\pi: S \rightarrow \mathbb{P}^{1}$ which do not meet the directrix $\sigma$. The resulting surface is $\mathbb{F}_{m}$, with $\sigma$ being the directrix. The image of $C$ under the contraction $S \rightarrow \mathbb{F}_{m}$ is the associated scroll construction.

For a vector bundle $E=\mathcal{O}\left(a_{1}\right) \oplus \cdots \oplus \mathcal{O}\left(a_{n}\right)$ on $\mathbb{P}^{1}$, set

$$
\lfloor E\rfloor=\min \left\{a_{i}\right\} \quad \text { and }\lceil E\rceil=\max \left\{a_{i}\right\} .
$$

Given a cover $\alpha: C \rightarrow \mathbb{P}^{1}$, the associated scroll construction $v: C \rightarrow \mathbb{F}_{m}$ can be made for $m \geq\left\lfloor E_{\alpha}\right\rfloor$. Conversely, given a point $\bar{C} \in \mathcal{V}_{g}^{\text {irr }}\left(\mathbb{F}_{m}, d \tau\right)$, let $C \rightarrow \bar{C}$ be the normalization. Then the induced cover $\alpha: C \rightarrow \mathbb{P}^{1}$ has $\left\lfloor E_{\alpha}\right\rfloor \leq m$.

Proposition 2.6. If $\tilde{M}(E)$ is nonempty, then

$$
\lceil E\rceil \leq \frac{2 g+2 d-2}{d} .
$$

Furthermore, if $E_{\alpha}$ comes from a cover $\left[\alpha: C \rightarrow \mathbb{P}^{1}\right]$, with $C$ irreducible, and where $\alpha$ does not factor nontrivially, then

$$
\frac{g+d-1}{\left(\begin{array}{l}
d \\
2
\end{array}\right)} \leq\left\lfloor E_{\alpha}\right\rfloor \leq \frac{g+d-1}{d-1} .
$$

Proof. The resolution of $\mathcal{O}_{C}$ in Theorem 1.4 tells us that $C \subset \mathbb{P} E_{\alpha}$ is not contained in any hyperplane divisor. Let $h$ denote the hyperplane divisor class associated to $\mathcal{O}_{\mathbb{P} E_{\alpha}}(1)$, and let $f$ denote the class of the fiber of $\pi: \mathbb{P} E \rightarrow \mathbb{P}^{1}$. Set $N:=\left\lceil E_{\alpha}\right\rceil$. Then the divisor class $h-N f$ is effective. Since $C$ is irreducible and does not lie in $(h-N f)$, it intersects $(h-N f)$ nonnegatively. Since $h \cdot[C]=2 g+2 d-2$ and $f \cdot[C]=d$, we conclude that $N \leq(2 d+2 g-2) / d$.

For the second inequality, we appeal to the associated scroll construction. Let $n:=\left\lfloor E_{\alpha}\right\rfloor$. Since $\alpha$ does not factor, $v: C \rightarrow \mathbb{F}_{n}$ must be birational onto its image. 
Adjunction on $\mathbb{F}_{n}$ gives

$$
p_{a}(v(C))=\left(\begin{array}{l}
d \\
2
\end{array}\right) n-(d-1) .
$$

The second statement now follows from the inequality $g \leq p_{a}(v(C))$.

The following result places a strong restriction on a large class of Tschirnhausen bundles $E$.

Proposition 2.7 [Ohbuchi 1997]. Let $\alpha: C \rightarrow \mathbb{P}^{1}$ be a cover of degree $d$, with $C$ irreducible, and where $\alpha$ does not factor nontrivially. Write $E_{\alpha}=\mathcal{O}\left(a_{1}\right) \oplus \cdots \oplus$ $\mathcal{O}\left(a_{d-1}\right)$, where $\left\lfloor E_{\alpha}\right\rfloor=a_{1} \leq a_{2} \leq \cdots \leq a_{d-1}=\left\lceil E_{\alpha}\right\rceil$. Then

$$
a_{i+1}-a_{i} \leq\left\lfloor E_{\alpha}\right\rfloor \text { for } 1 \leq i \leq d-2 .
$$

Remark 2.8. Proposition 2.7 implies the second inequality in Proposition 2.6.

Definition 2.9. We call a vector bundle $E$ on $\mathbb{P}^{1}$ of rank $d-1$ and degree $g+d-1$ tame if it satisfies inequalities (2-1), (2-2), and (2-3).

Notice that Proposition 2.6 and Proposition 2.7 imply that $E_{\alpha}$ is tame in the following two cases: $\alpha$ is simply branched, or $d$ is prime. Indeed, in either case, the cover cannot factor nontrivially.

Denote by $\leadsto$ the partial order on isomorphism classes of vector bundles on $\mathbb{P}^{1}$ given by $E \leadsto E^{\prime}$ if $E$ specializes to $E^{\prime}$ in a flat family. Note that isomorphism classes of vector bundles of rank $r$ and degree $n$ on $\mathbb{P}^{1}$ can be identified with unordered partitions of $n$ with $r$ parts. Then the order $\leadsto$ is the usual dominance order of partitions. For example, we have $(2,3,4) \leadsto(2,2,5)$ and $(2,3,4) \leadsto(1,4,4)$, but $(2,2,5)$ and $(1,4,4)$ are incomparable.

Define the finite set $\mathcal{T}[m]$ by $\mathcal{T}[m]:=\{$ Isomorphism classes of tame bundles $E$ of rank $d-1$,

$$
\text { degree } g+d-1 \text {, and }\lfloor E\rfloor=m\} \text {. }
$$

Observe that $\mathcal{T}[m]$ contains an element $E[m]$ such that $E[m] \leadsto E$ for all $E \in \mathcal{T}[m]$. In other words, $E[\mathrm{~m}]$ is the most generic among all the bundles in $\mathcal{T}[\mathrm{m}]$.

Theorem 2.10. Let $m \in \mathbb{Z}$ satisfy $(g+d-1) /\left(\begin{array}{c}d \\ 2\end{array}\right) \leq m \leq(g+d-1) /(d-1)$.

(1) If $M(E)$ is nonempty, then $E$ is a tame bundle.

(2) If $\lfloor E\rfloor \leq m$ then $M(E) \subset M(E[m])$.

(3) $M(E[m]) \subset M(E[m+1])$ for all $m$.

(4) $M(E[m])$ is an irreducible subvariety of $\mathcal{H}_{d, g}^{\dagger}$ of codimension $g-(d-1) m+1$ unless $m=\lfloor(g+d-1) /(d-1)\rfloor$, in which case $M(E[m])=\mathcal{H}_{d, g}^{\dagger}$. 
(5) If $d$ is prime, then all the statements above hold with $M(-)$ replaced by $\tilde{M}(-)$ and $\mathcal{H}_{d, g}^{\dagger}$ replaced by $\tilde{\mathcal{H}}_{d, g}^{\dagger}$.

In the proof, we use the following result (restated here for our setup):

Theorem 2.11 [Coppens 1999]. For all $m$ satisfying $(g+d-1) /\left(\begin{array}{c}d \\ 2\end{array}\right) \leq m \leq$ $(g+d-1) /(d-1)$, there is a genus- $g$ and degree-d cover $C \rightarrow \mathbb{P}^{1}$ with Tschirnhausen bundle $E[\mathrm{~m}]$. Moreover, $C$ is birational onto its image under the associated scroll construction $C \rightarrow \mathbb{F}_{m}$.

Proof of Theorem 2.10. The first statement follows from Propositions 2.6 and 2.7.

Before we proceed, we make two observation about the normalization $C$ of a general point $[\bar{C}]$ of $\mathcal{V}_{g}\left(\mathbb{F}_{m}, d \tau\right)$. First, $C \rightarrow \mathbb{P}^{1}$ is simply branched. Second, $C \rightarrow \mathbb{P}^{1}$ has Tschirnhausen bundle $E[m]$. Indeed, both are open conditions on $\mathcal{V}_{g}\left(\mathbb{F}_{m}, d \tau\right)$. By Proposition 2.4, there is a point satisfying the first condition. By Theorem 2.11, there is a point satisfying the second condition. By the irreducibility of $\mathcal{V}_{g}\left(\mathbb{F}_{m}, d \tau\right)$, a generic point satisfies both conditions.

For the second statement, suppose $\lfloor E\rfloor \leq m$ and let $\left[\alpha: C \rightarrow \mathbb{P}^{1}\right]$ be a point of $M(E)$. The associated scroll construction gives $v: C \rightarrow \mathbb{F}_{m}$; let $\bar{C} \subset \mathbb{F}_{m}$ be the image. Since $\alpha$ is simply branched, $v: C \rightarrow \bar{C}$ is birational. By the previous paragraph, we know that $[\bar{C}] \in \mathcal{V}_{g}\left(\mathbb{F}_{m}, d \tau\right)$ is the limit of an arc in $\mathcal{V}_{g}\left(\mathbb{F}_{m}, d \tau\right)$ whose general point corresponds to a curve with Tschirnhausen bundle $E[m]$. More precisely, we know that over a germ of a smooth curve (or the spectrum of a DVR) $\Delta$ there exists $\overline{\mathcal{C}} \subset \mathbb{F}_{m} \times \Delta$ such that:

- $\overline{\mathcal{C}} \rightarrow \Delta$ is a family of reduced and irreducible curves of geometric genus $g$.

- The fibers $\overline{\mathcal{C}}_{t} \subset \mathbb{F}_{m}$ are in the linear system $|d \tau|$.

- The special fiber $\overline{\mathcal{C}}_{0}$ is $\bar{C}$.

- The general fiber $\overline{\mathcal{C}}_{t}$ has the property that $\left(\overline{\mathcal{C}}_{t}\right)^{v} \rightarrow \mathbb{P}^{1}$ has Tschirnhausen bundle $E[m]$, where the superscript $v$ denotes normalization.

Let $\mathcal{C} \rightarrow \overline{\mathcal{C}}$ be the normalization of the total space of this family. The main theorem of [Teissier 1980] says that the fibers of $\mathcal{C} \rightarrow \Delta$ are the normalizations of the corresponding fibers of $\overline{\mathcal{C}} \rightarrow \Delta$. Considering the composition $\mathcal{C} \rightarrow \mathbb{P}^{1}$ of the sequence of maps $\mathcal{C} \rightarrow \overline{\mathcal{C}} \rightarrow \mathbb{F}_{m} \rightarrow \mathbb{P}^{1}$, we see that $\alpha: C \rightarrow \mathbb{P}^{1}$ is the limit of covers $\mathcal{C}_{t} \rightarrow \mathbb{P}^{1}$ which have Tschirnhausen bundle $E[m]$. The second statement follows.

The third statement is a corollary of the second statement.

For the fourth statement, suppose $m=\lfloor(g+d-1) /(d-1)\rfloor$. Then $E[m]$ is balanced, so $M(E[m])=\mathcal{H}_{d, g}^{\dagger}$. Suppose $m<\lfloor(g+d-1) /(d-1)\rfloor$. Let $U \subset \mathcal{V}_{g}\left(\mathbb{F}_{m}, d \tau\right)$ be the locus of nodal curves of geometric genus $g$ whose normalization is simply branched over $\mathbb{P}^{1}$. Then $U$ is a smooth open subset of $\mathcal{V}_{g}\left(\mathbb{F}_{m}, d \tau\right)$. Normalization of the universal family of curves in $\mathbb{F}_{m}$ of geometric genus $g$ gives a family of smooth curves of genus $g$ with a simply branched map of degree $d$ to $\mathbb{P}^{1}$ 
(induced from $\left.\mathbb{F}_{m} \rightarrow \mathbb{P}^{1}\right)$. By definition, the image is in $M(E[m])$. We thus get a dominant map

$$
q: U \rightarrow M(E[m]) .
$$

The fiber of $q$ over $\left[\alpha: C \rightarrow \mathbb{P}^{1}\right]$ corresponds to the global sections of $\mathcal{O}_{C}(m)$ that project nontrivially onto $E^{\vee}(m)$. For general $\alpha \in M(E[m])$, we have $E_{\alpha}=E[m]$. Also, since $m<\lfloor(g+d-1) /(d-1)\rfloor$, the bundle $E[m]$ has a unique $\mathcal{O}(m)$ summand and all other summands have degree greater than $m$. Therefore, the general fiber of $q$ has dimension $m+2$. From the dimension of $\mathcal{V}_{g}\left(\mathbb{F}_{m}, d \tau\right)$, we get

$$
\operatorname{dim} M(E[m])=\operatorname{dim} \mathcal{V}_{g}\left(\mathbb{F}_{m}, d \tau\right)-(m+2)=(d-1) m+g+2 d-3 .
$$

Since $\operatorname{dim} \mathcal{H}_{d, g}^{\dagger}=2 g+2 d-2$, the fourth statement follows.

For the last statement, all the arguments hold for $\tilde{M}(E)$ if $d$ is prime, since the associated scroll construction $v: C \rightarrow \mathbb{F}_{m}$ is automatically birational onto its image.

Theorem 2.10 gives us good control on the dimensions of the Maroni loci for $E$ based on the minimal summand of $E$. We must now consider those $E$ which are nongeneric, but nonetheless have the same minimal summand as the generic Tschirnhausen bundle. Set $k=\lfloor(g+d-1) /(d-1)\rfloor$. Then

$$
E[k]=\mathcal{O}(k)^{\oplus r} \oplus \mathcal{O}(k+1)^{\oplus d-r-1},
$$

where $0<r \leq d-1$. A general cover $\alpha \in \mathcal{H}_{d, g}^{\dagger}$ has $E[k]$ as its Tschirnhausen bundle. Let $E^{\prime}$ be any tame bundle, and set $s:=h^{0}\left(E^{\prime \vee}(k)\right)$. Upper-semicontinuity implies $s \geq r$. Suppose $s>r$. Define

$$
M^{\circ}\left(E^{\prime}\right)=\left\{\alpha \in \mathcal{H}_{d, g}^{\dagger} \mid E_{\alpha} \cong E^{\prime}\right\} .
$$

Then $M^{\circ}\left(E^{\prime}\right)$ is locally closed, and $\overline{M^{\circ}\left(E^{\prime}\right)}=M\left(E^{\prime}\right)$.

Lemma 2.12. Under the assumptions above, let $Z \subset M^{\circ}\left(E^{\prime}\right)$ be any irreducible component. Then the codimension of $\bar{Z}$ in $\mathcal{H}_{d, g}^{\dagger}$ is at least $(s-r)+1$.

Proof. Let $z=\operatorname{dim} Z$. We use the associated scroll construction over $Z$. We have an open subset $U$ of a vector bundle of rank $s+k+1$ over $Z$ and a morphism $U \rightarrow \mathcal{V}_{g}\left(\mathbb{F}_{k}, d \tau\right)$. Since $E^{\prime} \neq E[k]$, the closure of the image of $U$ is a proper subvariety of $\mathcal{V}_{g}\left(\mathbb{F}_{k}, \tau\right)$. In particular, we have $\operatorname{dim} U<\operatorname{dim} \mathcal{V}_{g}\left(\mathbb{F}_{k}, d \tau\right)=d k+2 d+$ $g-1$. The lemma follows from this inequality.

We now have the tools to determine all the Maroni divisors.

Proposition 2.13. The Maroni locus $M(E) \subset \mathcal{H}_{d, g}$ is a divisor if and only if $g=(k-1)(d-1)$ for some integer $k \geq 1$, and

$$
E=E[k-1]=\mathcal{O}(k-1) \oplus \mathcal{O}(k)^{\oplus d-3} \oplus \mathcal{O}(k+1) .
$$

Furthermore, in this situation, $M(E[k-1])$ is irreducible. 
Proof. If $\lfloor E\rfloor=k=\lfloor(g+d-1) /(d-1)\rfloor$, then the statement follows by applying Lemma 2.12. If, on the other hand, $\lfloor E\rfloor<\lfloor(g+d-1) /(d-1)\rfloor$, then the statement follows from statement (4) of Theorem 2.10.

We record a particularly interesting case of the irreducibility of the Maroni divisor:

Corollary 2.14. Let $g=2(d-1)$. Then $M(E[2]) \subset \mathcal{H}_{d, g}$ is irreducible, and it is the ramification locus of the generically finite and dominant forgetful map $\mu: \mathcal{H}_{d, g} \rightarrow \mathcal{M}_{g}$.

Proof. The irreducibility statement follows from Theorem 2.10. To show that $M(E[2])$ is the ramification locus of $\mu$, consider $\left[\alpha: C \rightarrow \mathbb{P}^{1}\right] \in \mathcal{H}_{d, g}$ and the map of sheaves

$$
0 \longrightarrow \alpha^{*}\left(T_{\mathbb{P} 1}\right) \longrightarrow T_{C} \longrightarrow N_{\alpha} \longrightarrow 0 .
$$

The tangent space to $\mathcal{H}_{d, g}$ at $\alpha$ is $H^{0}\left(C, N_{\alpha}\right) / \alpha^{*} H^{0}\left(\mathbb{P}^{1}, T_{\mathbb{P} 1}\right)$ and the tangent space to $\mathcal{M}_{g}$ at $C$ is $H^{1}\left(C, T_{C}\right)$. The map

$$
d \mu: H^{0}\left(C, N_{\alpha}\right) / \alpha^{*} H^{0}\left(\mathbb{P}^{1}, T_{\mathbb{P}^{1}}\right) \rightarrow H^{1}\left(C, T_{C}\right)
$$

fails to be surjective precisely when $H^{1}\left(C, \alpha^{*} T_{\mathbb{P} 1}\right) \neq 0$, i.e., when $\alpha \in M(E[2])$.

2B. Linear independence of $T, D$, and $\Delta$. In this section, we prove that the divisorial components of the boundary of $\tilde{\mathcal{H}}_{d, g}$ are linearly independent. Define the closed loci $T, D, \Delta$ in $\widetilde{\mathcal{H}}_{d, g}$ by

$$
\begin{aligned}
& T=\overline{\left\{\left[\alpha: C \rightarrow \mathbb{P}^{1}\right] \mid \alpha^{-1}(q)=3 p_{1}+p_{2}+\cdots+p_{d-2} \text { for some } q \text { and distinct } p_{i} \cdot\right\}} \\
& D=\overline{\left\{\left[\alpha: C \rightarrow \mathbb{P}^{1}\right] \mid \alpha^{-1}(q)=2 p_{1}+2 p_{2}+p_{3}+\cdots+p_{d-2}\right.}
\end{aligned}
$$

for some $q$ and distinct $\left.p_{i} \cdot\right\}$

$$
\Delta=\overline{\left\{\left[\alpha: C \rightarrow \mathbb{P}^{1}\right] \mid C \text { is singular. }\right\}}
$$

These three loci correspond to the three possibilities of the limit when two branch points of a branched cover come together. Note that $T, D$, and $\Delta$ are irreducible and their union is the complement of $\mathcal{H}_{d, g}$ in $\tilde{\mathcal{H}}_{d, g}$.

Proposition 2.15. For $d \geq 4$, the classes of $T, D$, and $\Delta$ are linearly independent in $\operatorname{Pic}_{\mathbb{Q}}\left(\tilde{\mathcal{H}}_{d, g}\right)$. For $d \geq 3$, the same is true for the classes of $T$ and $\Delta$.

Proof. We construct curves with nonsingular intersection matrix with our divisors. For this, a slight enlargement of $\widetilde{\mathcal{H}}_{d, g}$ is more convenient. Define $\widetilde{\mathcal{H}}_{d, g}^{n s}$ as the moduli space of $\left[\alpha: C \rightarrow \mathbb{P}^{1}\right]$, where $C$ is an at worst nodal curve of arithmetic genus $g$, not necessarily irreducible, but without any separating nodes, and $\alpha$ is a map of degree $d$. The target $\mathbb{P}^{1}$ is taken to be unframed. It is easy to see that $\widetilde{\mathcal{H}}_{d, g}$ is a dense open subset of $\widetilde{\mathcal{H}}_{d, g}^{n s}$ with codimension-2 complement. Abusing notation, 


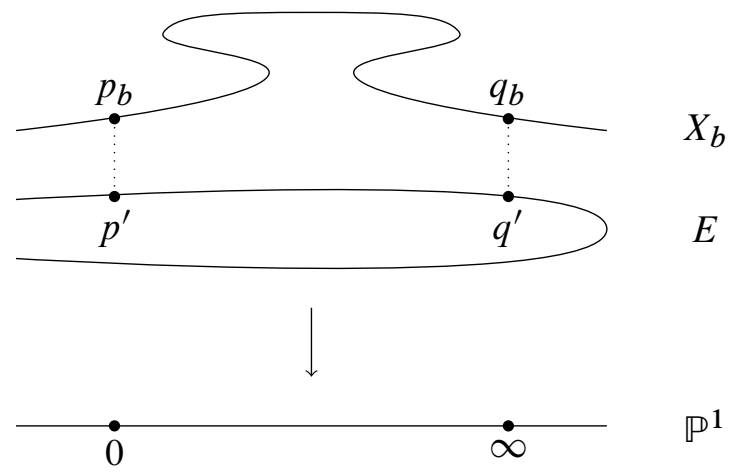

Figure 1. We construct families of covers parametrized by $b \in B$ by attaching a variable family of covers $\alpha_{b}: X_{b} \rightarrow \mathbb{P}^{1}$ to a fixed cover $\beta: E \rightarrow \mathbb{P}^{1}$.

we denote the closures of $T, D$, and $\Delta$ in $\widetilde{\mathcal{H}}_{d, g}^{n s}$ by the same letters. It suffices to prove the proposition for $\widetilde{\mathcal{H}}_{d, g}^{n s}$.

We now construct test curves in $\widetilde{\mathcal{H}}_{d, g}^{n s}$. Pick nonnegative integers $g_{1}$ and $g_{2}$ with $g_{1}+g_{2}=g-1$ and positive integers $d_{1}$ and $d_{2}$ with $d_{1}+d_{2}=d$. Take a family $\alpha_{b}: X_{b} \rightarrow \mathbb{P}^{1}$ of covers of degree $d_{1}$ and genus $g_{1}$, where $b$ denotes a parameter on a smooth complete curve $B$. Assume that we have two sections $p, q: B \rightarrow X$ with $\alpha_{b}\left(p_{b}\right)=0$ and $\alpha_{b}\left(q_{b}\right)=\infty$ for all $b \in B$. Take $\beta: E \rightarrow \mathbb{P}^{1}$ to be a fixed simply branched cover of degree $d_{2}$ and genus $g_{2}$, unramified over 0 and $\infty$, and let $p^{\prime}, q^{\prime} \in E$ be two points over 0 and $\infty$ respectively. Our test curve in $\widetilde{\mathcal{H}}_{d, g}^{n s}$ is given by the family $\gamma_{b}: C_{b} \rightarrow \mathbb{P}^{1}$, where $C_{b}$ is obtained by gluing $\left(X_{b}, p_{b}, q_{b}\right)$ to the constant family $\left(E, p^{\prime}, q^{\prime}\right)$, and $\gamma_{b}: C_{b} \rightarrow \mathbb{P}^{1}$ is induced from $\alpha: X_{b} \rightarrow \mathbb{P}^{1}$ and $\beta: E \rightarrow \mathbb{P}^{1}$. The construction is depicted in Figure 1.

Let $T_{\alpha}, D_{\alpha}$, and $\Delta_{\alpha}$ denote the pullbacks of the divisor classes $T, D$, and $\Delta$ along the map from $B$ to $\widetilde{\mathcal{H}}_{d_{1}, g_{1}}$ given by $\alpha_{b}$. Define $T_{\gamma}, D_{\gamma}$, and $\Delta_{\gamma}$ likewise. Let $e$ be the intersection number of $\operatorname{Br}(\alpha)$ with a horizontal section of $\mathbb{P}^{1} \times B$. Denote by $[p]$ and $[q]$ respectively the classes of $p(B)$ and $q(B)$ on $X$.

Claim. With the notation above, we have

$$
\begin{aligned}
\operatorname{deg} T_{\gamma} & =\operatorname{deg} T_{\alpha}+3([p]+[q]) \cdot \operatorname{Ram}(\alpha), \\
\operatorname{deg} D_{\gamma} & =\operatorname{deg} D_{\alpha}+\left(2 g_{2}+2 d_{2}-2\right) e+4 e-4([p]+[q]) \cdot \operatorname{Ram}(\alpha), \text { and } \\
\operatorname{deg} \Delta_{\gamma} & =\operatorname{deg} \Delta_{\alpha}+[p]^{2}+[q]^{2} .
\end{aligned}
$$

Proof of the claim. The pullback of the line bundle $\mathcal{O}(\Delta)$ from $\tilde{\mathcal{H}}_{d, g}^{n s}$ to $B$ is given by

$$
\left(N_{p / X} \otimes N_{p^{\prime} / E}\right) \otimes\left(N_{q / E} \otimes N_{q^{\prime} / E}\right) \otimes \mathcal{O}_{B}\left(\Delta_{\alpha}\right),
$$


where $N_{p / X}$ denotes the normal bundle of $p$ in $X$, and so on. The third equation follows.

For a generic $b \in B$, the point of $\tilde{\mathcal{H}}_{d, g}^{n s}$ given by $\gamma_{b}: C_{b} \rightarrow \mathbb{P}^{1}$ does not lie in $T$ or $D$. We have the following specializations:

(1) $\alpha_{b}: X_{b} \rightarrow \mathbb{P}^{1}$ has a fiber of the form $3 p_{1}+p_{2}+\cdots$. Such $b$ are precisely the points of $T_{\alpha}$, each contributing 1 to $\operatorname{deg} T_{\gamma}$.

(2) $\alpha_{b}: X_{b} \rightarrow \mathbb{P}^{1}$ has a fiber of the form $2 p_{1}+2 p_{2}+p_{3}+\cdots$. Such $b$ are precisely the points of $D_{\alpha}$, each contributing 1 to $\operatorname{deg} D_{\gamma}$

(3) A branch point of $\alpha_{b}: X_{b} \rightarrow \mathbb{P}^{1}$ coincides with a branch point of $\beta: E \rightarrow \mathbb{P}^{1}$. There are $\left(2 g_{2}+2 d_{2}-2\right) e$ such $b$, each contributing 1 to $\operatorname{deg} D_{\gamma}$.

(4) $p_{b}$ (resp. $q_{b}$ ) is a ramification point of $\alpha_{b}$. We compute the intersection multiplicity of $B$ with $T$ and $D$ at such a point by looking at a versal deformation space of $\gamma_{b}$. We may restrict $\gamma_{b}$ over an analytic neighborhood $U$ of 0 (resp. $\infty$ ). Let $x$ be a coordinate on $U$. Then $\gamma_{b}^{-1}(U) \rightarrow U$ has the form

$$
U[y] /\left(y^{3}-x y\right) \sqcup U \sqcup \cdots \sqcup U \rightarrow U .
$$

A versal deformation of this cover is given over $\operatorname{Spec} \mathbb{C}[s, t]$ by

$$
U[y] /\left(y^{3}-x y-s x-t\right) \sqcup U \sqcup \cdots \sqcup U \rightarrow U .
$$

In Spec $\mathbb{C}[s, t]$, the divisor $D$ does not contain the origin, and hence the intersection number of $B$ with $D$ at $b$ is 0 . The divisor $T \subset \operatorname{Spec} \mathbb{C}[s, t]$ is defined by $t=0$. The curve $B$ approaches the origin along the locus where $U[y] /\left(y^{3}-x y-s x-t\right)$ is singular, namely along $s^{3}+t=0$. We deduce that the intersection number of $B$ with $T$ at $b$ is 3. There are $[p] \cdot \operatorname{Ram}(\alpha)(\operatorname{resp}$. $[q] \cdot \operatorname{Ram}(\alpha)) \operatorname{such} b$, each contributing 3 to $\operatorname{deg} T_{\gamma}$.

(5) $p_{b}$ (resp. $q_{b}$ ) is not a ramification point of $\alpha_{b}$, but lies over a branch point. Again, we look at a versal deformation of $\gamma_{b}$. In this case, $\gamma_{b}^{-1}(U) \rightarrow U$ has the form

$$
U[y] /\left(y^{2}-x\right) \sqcup U[z] /\left(z^{2}-x^{2}\right) \sqcup U \sqcup \cdots \sqcup U \rightarrow U .
$$

A versal deformation of this cover is given over $\operatorname{Spec} \mathbb{C}[s, t]$ by

$$
U[y] /\left(y^{2}-x\right) \sqcup U[z] /\left(z^{2}-x^{2}-s x-t\right) \sqcup U \sqcup \cdots \sqcup U \rightarrow U .
$$

In Spec $\mathbb{C}[s, t]$, the divisor $T$ does not contain the origin, and hence the intersection number of $B$ with $T$ at $b$ is 0 . The divisor $D \subset \operatorname{Spec} \mathbb{C}[s, t]$ is defined by $t=0$. The curve $B$ approaches the origin along the locus where $U[z] /\left(z^{2}-x^{2}-s x-t\right)$ is singular, namely along $s^{2}-4 t=0$. We deduce that the intersection number of $B$ with $D$ at $b$ is 2 . Let us count the number of such points, first for $p_{b}$, and analogously for $q_{b}$. The points $b$ for which $p_{b}$ is not a ramification point but lies over a branch point correspond to the intersection points of $\operatorname{Br}(\alpha) \cap\{0\} \times B$ which are not the 
images of the points of $\operatorname{Ram}(\alpha) \cap p(B)$. Note, however, that the image of a point of $\operatorname{Ram}(\alpha) \cap p(B)$ is actually a point of tangency of $\operatorname{Br}(\alpha)$ with $\{0\} \times B$, and hence contributes 2 to the intersection number $e=\operatorname{Br}(\alpha) \cdot\{0\} \times B$. The remaining count, which we want, is therefore $e-2[p] \cdot \operatorname{Ram}(\alpha)$. Similarly, the count for $q_{b}$ is $e-2[q] \cdot \operatorname{Ram}(\alpha)$.

The expressions for $T_{\gamma}$ and $D_{\gamma}$ follow from combining the above contributions.

Returning to the proof of the proposition, consider the following three particular test curves for $d \geq 4$.

$B_{1}$ : Take $\alpha_{b}: X_{b} \rightarrow \mathbb{P}^{1}$ to be a family of hyperelliptic curves of genus $g-1$ obtained by taking a double cover $X \rightarrow \mathbb{P}^{1} \times \mathbb{P}^{1}$ branched along a curve of type $(2 g, 2)$. To have sections $p$ and $q$ of $X$ over $\{0\} \times \mathbb{P}^{1}$ and $\{\infty\} \times \mathbb{P}^{1}$, let the branch divisor be tangent to $\{0\} \times \mathbb{P}^{1}$ and $\{\infty\} \times \mathbb{P}^{1}$. Take $E$ to be a smooth rational curve and $\gamma: E \rightarrow \mathbb{P}^{1}$ a generic cover of degree $d-2$.

$B_{2}$ : Take $\alpha_{b}: X_{b} \rightarrow \mathbb{P}^{1}$ to be a family of trigonal curves of genus $g-1$ obtained by taking a general pencil on $\mathbb{F}_{0}$ in the linear system $|((g+1) / 2,3)|$ if $g$ is odd, or on $\mathbb{F}_{1}$ in the linear system $\mid 3$ - directrix $+(g / 2+2)$ - fiber $\mid$ if $g$ is even. Two base-points give $p_{b}$ and $q_{b}$. Take $E$ to be a rational curve and $\gamma: E \rightarrow \mathbb{P}^{1}$ a general cover of degree $d-3$.

$B_{3}$ : Take $\alpha_{b}: X_{b} \rightarrow \mathbb{P}^{1}$ to be a family of hyperelliptic curves of genus $g-2$ as in $B_{1}$. Take $E$ to be a smooth genus-1 curve and $\gamma: E \rightarrow \mathbb{P}^{1}$ a generic cover of degree $d-2$. This curve exists only for $d \geq 4$.

Using the claim, we get the following nonsingular intersection matrix:

\begin{tabular}{c|rll} 
& $T$ & \multicolumn{1}{c}{$D$} & \multicolumn{1}{c}{$\Delta$} \\
\hline$B_{1}$ & 6 & $4 d-12$ & $8 g-6$ \\
$B_{2}$ & $3 g+9$ & $8 d-24$ & $7 g-3$ \\
$B_{3}$ & 6 & $4 d-8$ & $8 g-14$
\end{tabular}

For $d=3$, we take a pencil in $\mathbb{F}_{0}$ or $\mathbb{F}_{1}$ as in $B_{1}$, but of trigonal curves of genus $g$, without any $E$. Then the middle column vanishes, and the second row becomes $(3 g+6,0,7 g+6)$, which is linearly independent from the first row.

\section{Degree 3}

Let $C$ be a curve of genus $g$ and $\alpha: C \rightarrow \mathbb{P}^{1}$ a map of degree 3 . The relative canonical map embeds $C$ as a divisor in a $\mathbb{P}^{1}$-bundle $\mathbb{P} E$ over $\mathbb{P}^{1}$, where $E$ is a vector bundle of rank 2 and degree $g+2$.

Let

$$
E^{\text {gen }}=\mathcal{O}\left(\left\lfloor\frac{g+2}{2}\right\rfloor\right) \oplus \mathcal{O}\left(\left\lceil\frac{g+2}{2}\right\rceil\right)
$$


be the most generic vector bundle on $\mathbb{P}^{1}$ of rank 2 and degree $g+2$. Set

$$
U_{E^{\text {gen }}}:=\left\{\alpha \in \tilde{\mathcal{H}}_{3, g} \mid E_{\alpha} \cong E^{\text {gen }}\right\} .
$$

Note that $U_{E \text { gen }}$ is an open subset of $\widetilde{\mathcal{H}}_{3, g}$.

Proposition 3.1. The complement of $U_{E^{\text {gen }}}$ in $\widetilde{\mathcal{H}}_{3, g}$ is a divisor if and only if $g$ is even, in which case it is irreducible.

Proof. This is the degree-3 case of Proposition 2.13.

Let $\pi: \mathbb{P} E^{\text {gen }} \rightarrow \mathbb{P}^{1}$ be the projection. Set

$$
V=H^{0}\left(\mathbb{P}^{1}, \operatorname{Sym}^{3} E^{\text {gen }} \otimes \operatorname{det} E^{\text {gen } \vee}\right) .
$$

Elements of $\mathbb{P}_{\text {sub }} V$ correspond to divisors in the linear series of the line bundle $\mathcal{O}_{\mathbb{P} E} E_{\text {gen }}(3) \otimes \pi^{*}\left(\operatorname{det} E^{\text {gen }}\right)^{\vee}$ on $\mathbb{P} E^{\text {gen }}$. Let $C_{v} \subset \mathbb{P} E^{\text {gen }}$ be the divisor corresponding to $v \in V$. Let $V^{\circ} \subset \mathbb{P}_{\text {sub }} V$ be the open locus consisting of $v \in V^{\circ}$ for which $C_{v}$ is irreducible and at worst nodal. Let $G:=\operatorname{Aut}(\pi)$ be the group of automorphisms of $\mathbb{P} E^{\text {gen }}$ over $\mathbb{P}^{1}$. Then $G$ acts on $V^{\circ}$. The assignment

$$
v \mapsto\left[\pi: C_{v} \rightarrow \mathbb{P}^{1}\right]
$$

gives a map

$$
q: V^{\circ} \rightarrow \tilde{\mathcal{H}}_{3, g}^{\dagger}
$$

Denote by $U_{E \text { gen }}^{\dagger}$ the preimage of $U_{E^{\text {gen }}}$ under $\widetilde{\mathcal{H}}_{3, g}^{\dagger} \rightarrow \widetilde{\mathcal{H}}_{3, g}$.

Proposition 3.2. The image of $q$ is $U_{E \text { gen }}^{\dagger}$. The fibers of $q$ consist of single G-orbits. Proof. For brevity, set $E=E^{\text {gen }}$. For $v \in V^{\circ}$, consider the sequence

$$
0 \longrightarrow \mathcal{O}_{\mathbb{P} E}(-3) \otimes \pi^{*} \operatorname{det} E \longrightarrow \mathcal{O}_{\mathbb{P} E} \longrightarrow \mathcal{O}_{C_{v}} \longrightarrow 0 .
$$

Applying $R \pi_{*}$, we get

$$
0 \longrightarrow \mathcal{O}_{\mathbb{P} 1} \longrightarrow \pi_{*} \mathcal{O}_{C_{u}} \longrightarrow E^{\vee} \longrightarrow 0,
$$

which says that the Tschirnhausen bundle of $C_{u} \rightarrow \mathbb{P}^{1}$ is $E$. Conversely, from the Casnati-Ekedahl resolution, it follows that every point of $U_{E^{\text {gen }}}^{\dagger}$ is in the image of $q$.

Let $u, v \in U_{E \text { gen }}^{\dagger}$ be in a fiber of $q$. Then there is an isomorphism $C_{u} \rightarrow C_{v}$ over the identity of $\mathbb{P}^{1}$. The sequence (3-1) for $C_{u}$ and $C_{v}$ shows that such an isomorphism induces an isomorphism $E \rightarrow E$. The induced automorphism of $\mathbb{P} E$ over $\mathbb{P}^{1}$ takes $C_{u}$ to $C_{v}$ and hence $u$ to $v$.

Proposition 3.3 (Picard rank conjecture for degree 3). We have $\mathrm{Pic}_{\mathbb{Q}} \mathcal{H}_{3, g}=0$. 
Proof. Retain the notation introduced above. For brevity, set $U=U_{E}$ gen and $U^{\dagger}=U_{E}^{\dagger}$.en. Then $V^{\circ} \rightarrow U^{\dagger}$ is a quotient by $G$ and $U^{\dagger} \rightarrow U$ is a quotient by $\mathrm{PGL}_{2}$. By Proposition 1.2 and Proposition 3.2, we have

$$
\begin{aligned}
\operatorname{rkPic} \operatorname{PQ}_{\mathbb{Q}} U \leq \operatorname{rkPic}_{\mathbb{Q}} U^{\dagger}+\operatorname{rk} \chi\left(\operatorname{PGL}_{2}\right) & =\operatorname{rkPic}_{\mathbb{Q}} U^{\dagger} \\
& \leq \operatorname{rkPic}_{\mathbb{Q}} V^{\circ}+\operatorname{rk} \chi(G) \leq 1+\operatorname{rk} \chi(G) .
\end{aligned}
$$

The final inequality follows because $V^{\circ}$ is an open subset of a projective space. Let $e$ be the number of divisorial components of $\widetilde{\mathcal{H}}_{3, g} \backslash U$. We then get the bound

$$
\operatorname{rkPic} \widetilde{\mathcal{H}}_{3, g} \leq \operatorname{rkPic}_{\mathbb{Q}} U+e \leq 1+\operatorname{rk} \chi(G)+e .
$$

If $g$ is even, then

$$
\begin{aligned}
G & =\mathrm{PGL}_{2}, \\
\operatorname{rk} \chi(G) & =0, \\
e & =1 \quad \text { by Proposition 3.1. }
\end{aligned}
$$

If $g$ is odd, then

$$
\begin{aligned}
G & =\left\{\left(\begin{array}{ll}
a & l \\
0 & b
\end{array}\right) \mid a, b \in \mathbb{C}^{*}, l \in H^{0}\left(\mathbb{P}^{1}, \mathcal{O}(1)\right)\right\} / \mathbb{C}^{*}, \\
\operatorname{rk} \chi(G) & =1, \\
e & =0 \text { by Proposition } 3.1 .
\end{aligned}
$$

In either case, we have

$$
\operatorname{rkPic} \tilde{\mathcal{H}}_{3, g} \leq 2 .
$$

By Proposition 2.15, the classes in $\operatorname{Pic}_{\mathbb{Q}}\left(\widetilde{\mathcal{H}}_{3, g}\right)$ of the two components of $\widetilde{\mathcal{H}}_{3, g} \backslash \mathcal{H}_{3, g}$ are linearly independent. Therefore, we get $\operatorname{Pic}_{\mathbb{Q}} \mathcal{H}_{3, g}=0$ as desired.

\section{Degree 4}

Let $C$ be a curve of genus $g$ and $\alpha: C \rightarrow \mathbb{P}^{1}$ a map of degree 4 . The relative canonical map embeds $C$ into a $\mathbb{P}^{2}$-bundle $\mathbb{P} E$ over $\mathbb{P}^{1}$, where $E$ is a vector bundle of rank 3 and degree $g+3$. The Casnati-Ekedahl structure theorem provides the following resolution of $\mathcal{O}_{C}$ :

$$
0 \longrightarrow \pi^{*} \operatorname{det} E(-4) \longrightarrow \pi^{*} F(-2) \longrightarrow \mathcal{O}_{\mathbb{P} E} \longrightarrow \mathcal{O}_{C} \longrightarrow 0,
$$

where $F$ is a vector bundle of rank 2 and degree $g+3$.

Explicitly, we can describe $C \subset \mathbb{P} E$ as follows. Write $F=\mathcal{O}(a) \oplus \mathcal{O}(b)$, where $a+b=g+3$ and $a \leq b$. Let $h$ denote the divisor class associated to $\mathcal{O}_{\mathbb{P} E}(1)$ on $\mathbb{P} E$ and $f$ the class of the fiber of the projection $\pi: \mathbb{P} E \rightarrow \mathbb{P}^{1}$. Then the curve $C$ 
is the complete intersection of two divisors:

$$
C=Q_{a} \cap Q_{b},
$$

where $\left[Q_{a}\right]=2 h-a f$ and $\left[Q_{b}\right]=2 h-b f$.

We can describe the equations of $Q_{a}$ and $Q_{b}$ even more explicitly as follows. Write $E=\mathcal{O}\left(m_{1}\right) \oplus \mathcal{O}\left(m_{2}\right) \oplus \mathcal{O}\left(m_{3}\right)$. Over an open set $U \subset \mathbb{P}^{1}$, let $X, Y$, and $Z$ denote the relative coordinates on $\left.\mathbb{P} E\right|_{U}$ corresponding to the three summands of $E$. Assume that $m_{1} \leq m_{2} \leq m_{3}$. Over $U$, the divisor $Q_{a}$ is the zero locus of a form

$$
p_{1,1} X^{2}+p_{1,2} X Y+p_{1,3} X Z+p_{2,2} Y^{2}+p_{2,3} Y Z+p_{3,3} Z^{2},
$$

where $p_{i, j}$ is the restriction to $U$ of a global section of $\mathcal{O}\left(m_{i}+m_{j}-a\right)$. Similarly, over $U$, the divisor $Q_{b}$ is the zero locus of a form

$$
q_{1,1} X^{2}+q_{1,2} X Y+q_{1,3} X Z+q_{2,2} Y^{2}+q_{2,3} Y Z+q_{3,3} Z^{2},
$$

where $q_{i, j}$ is the restriction to $U$ of a global section of $\mathcal{O}\left(m_{i}+m_{j}-b\right)$.

The irreducibility of $C$ puts some restrictions on the possible $(E, F)$. Indeed, if $p_{1,1}=q_{1,1}=0$, then the section $[X: Y: Z]=[1: 0: 0]$ of $\mathbb{P} E$ is contained in both $Q_{a}$ and $Q_{b}$, making $C=Q_{a} \cap Q_{b}$ reducible. An irreducible $C$ thus forces

$$
2 m_{1} \geq a .
$$

Proposition 4.1. Let $E$ be a vector bundle of rank 3 and degree $g+3$ and $F$ a vector bundle of rank 2 and degree $g+3$. If the locus $M(E, F)$ is nonempty, then it is irreducible and unirational.

Proof. Consider the dense open subset $M^{\circ}(E, F) \subset M(E, F)$ corresponding to those $\alpha \in \mathcal{H}_{4, g}$ that have $E_{\alpha} \cong E$ and $F_{\alpha} \cong F$. It suffices to prove the statement for $M^{\circ}(E, F)$. Consider the vector space

$$
V:=H^{0}\left(\mathbb{P}^{1}, F^{\vee} \otimes \operatorname{Sym}^{2} E\right) .
$$

Elements of $V$ correspond to maps $\pi^{*} F(-2) \rightarrow \mathcal{O}_{\mathbb{P} E}$. Let $V^{\circ} \subset V$ be the open subset where the ideal generated by the image of $\pi^{*} F(-2)$ defines a smooth curve, simply branched over $\mathbb{P}^{1}$. Then $V^{\circ}$ surjects onto $M^{\circ}(E, F)$.

Remark 4.2. From the dominant map $V^{\circ} \rightarrow M(E, F)$ in the proof of Proposition 4.1 , it is easy to compute the codimension of $M(E, F)$ in $\mathcal{H}_{4, g}$, which is $\operatorname{codim} M(E, F)=\operatorname{dim}_{E_{x t}}{ }^{1}(E, E)+\operatorname{dim} \operatorname{Ext}^{1}(F, F)-\operatorname{dim}_{E_{x t}}{ }^{1}\left(F, \operatorname{Sym}^{2} F\right)$.

We may think of $\operatorname{dim} \operatorname{Ext}^{1}(E, E)+\operatorname{dim} \operatorname{Ext}^{1}(F, F)$ as the "expected codimension". The next example shows that the actual codimension is not always the expected codimension. 
Example 4.3. Let $E=\mathcal{O}(m) \oplus \mathcal{O}(2 m) \oplus \mathcal{O}(g+3-3 m)$, where $\lceil(g+3) / 6\rceil \leq$ $m<(g+3) / 5$. To get an irreducible curve $C$, the only possibility for $F$ is $F=\mathcal{O}(2 m) \oplus \mathcal{O}(g+3-2 m)$, by (4-3). The resulting locus $M(E, F)$ is not of expected codimension because $\operatorname{dim} \operatorname{Ext}^{1}\left(F, \operatorname{Sym}^{2} E\right)$ is nonzero.

Example 4.4. The Maroni locus $M(E)$ may be reducible. Let $g=12$, and consider the bundle $E=\mathcal{O}(3) \oplus \mathcal{O}(5) \oplus \mathcal{O}(7)$. Then the reader can easily check (using Bertini's theorem) that $M(E, F)$ and $M\left(E, F^{\prime}\right)$ are nonempty and of equal codimension $\operatorname{dim} \operatorname{Ext}^{1}(E, E)$ for the bundles $F=\mathcal{O}(6) \oplus \mathcal{O}(9)$ and $F^{\prime}=\mathcal{O}(5) \oplus \mathcal{O}(10)$. Therefore $M(E, F)$ and $M\left(E, F^{\prime}\right)$ are two components of $M(E)$. It is easy to see by analyzing the explicit equations that these are the only components of $M(E)$.

Let $E^{\text {gen }}$ (resp. $F^{\text {gen }}$ ) be the most generic vector bundle on $\mathbb{P}^{1}$ of rank 3 (resp. 2 ) and degree $g+3$. Define

$$
\begin{aligned}
U_{E^{\text {gen }}} & :=\left\{\alpha \in \widetilde{\mathcal{H}}_{4, g} \mid E_{\alpha} \cong E^{\text {gen }}\right\}, \\
U_{F^{\text {gen }}} & :=\left\{\alpha \in \widetilde{\mathcal{H}}_{4, g} \mid F_{\alpha} \cong F^{\text {gen }}\right\}, \\
U_{E^{\text {gen }}, F^{\text {gen }}} & :=U_{E^{\text {gen }}} \cap U_{F^{\text {gen }}} .
\end{aligned}
$$

It is easy to see that these are open subsets of $\widetilde{\mathcal{H}}_{d, g}$. Our next task is to identify the divisorial components of their complements.

Proposition 4.5. The subvariety $M:=\widetilde{\mathcal{H}}_{4, g} \backslash U_{E^{\text {gen }}}$ is a divisor if and only if $g$ is divisible by 3 , in which case it is irreducible.

Proof. This is the degree-4 case of Proposition 2.13.

For the complement of $U_{F}$ gen, we could do a careful analysis of the defining equations of $C$ in $\mathbb{P} E$, as we will have to do for the next case of $d=5$. But we can take a more geometric approach using the resolvent cubic construction. Originally due to Recillas [1973], the construction can be described as follows. For simplicity, we give an informal description, restricting to simply branched covers. See [Casnati 1998] for a detailed account. Consider a point $\left[\alpha: C \rightarrow \mathbb{P}^{1}\right]$ of $\mathcal{H}_{4, g}$. The resolution of $\mathcal{O}_{C}$ as an $\mathcal{O}_{\mathbb{P} E_{\alpha}}$-module shows that $C \subset \mathbb{P} E_{\alpha}$ is the complete intersection of two relative quadrics. A fiber of $\mathbb{P} F_{\alpha} \rightarrow \mathbb{P}^{1}$ naturally corresponds to the pencil of conics in the corresponding fiber of $\mathbb{P} E_{\alpha} \rightarrow \mathbb{P}^{1}$ containing the corresponding fiber of $C \rightarrow \mathbb{P}^{1}$. Each such pencil contains three singular conics, counted with multiplicity. The total locus of these singular conics forms a trigonal curve $R(C) \subset \mathbb{P} F_{\alpha}$. Let $R(\alpha): R(C) \rightarrow \mathbb{P}^{1}$ be the projection. We call $R(\alpha)$ the resolvent cubic of $\alpha$. Using that $C \rightarrow \mathbb{P}^{1}$ is simply branched, it is easy to check that $R(C)$ is smooth and the branch divisor of $R(\alpha)$ coincides with the branch divisor of $\alpha$. In particular, $R(C)$ has genus $g+1$. The association $\alpha \rightarrow R(\alpha)$ defines a map

$$
R: \mathcal{H}_{4, g} \rightarrow \mathcal{H}_{3, g+1}
$$


which we call the resolvent cubic map. The fiber of $R$ over a point $\left[D \rightarrow \mathbb{P}^{1}\right]$ in $\mathcal{H}_{3, g+1}$ corresponds bijectively to the set of étale double covers $D^{\prime} \rightarrow D$ (see [Recillas 1973], [Casnati 1998, Theorem 6.5], or [Donagi 1981]). In particular, $R$ is a finite morphism.

Proposition 4.6. Let $F$ be a vector bundle of rank 2 and degree $g+3$ on $\mathbb{P}^{1}$. The Casnati-Ekedahl locus $C(F) \subset \mathcal{H}_{4, g}$ is nonempty if and only if $\lfloor F\rfloor \geq\lceil(g+3) / 3\rceil$. In this case, it is of the expected codimension $\operatorname{dim} \operatorname{Ext}^{1}(F, F)$.

Proof. Consider a point $\left[\alpha: C \rightarrow \mathbb{P}^{1}\right]$ of $\mathcal{H}_{4, g}$ and its resolvent cubic $R(\alpha): R(C) \rightarrow$ $\mathbb{P}^{1}$. Since $R(C) \subset \mathbb{P} F_{\alpha}$, and $F_{\alpha}$ is a vector bundle of rank 2 and degree $(g+1)+2$, it must be the Tschirnhausen bundle of $R(C)$. That is, we have $E_{R(\alpha)}=F_{\alpha}$. By [Recillas 1973], the map $R$ is finite, and hence $C(F)=R^{-1}(M(F))$. Both statements about $C(F)$ now follow from the corresponding statements about $M(F)$.

Proposition 4.7. Let $g \geq 4$. The subvariety $C E:=\mathcal{H}_{4, g} \backslash U_{F}$ gen has codimension at least 2 if $g$ is even and is an irreducible divisor if $g$ is odd.

Proof. The image $R\left(U_{F \text { gen }}\right) \subset \mathcal{H}_{3, g+1}$ is the open locus of trigonal covers having $F^{\text {gen }}$ as their Tschirnhausen bundle. The complement $Z:=\mathcal{H}_{3, g+1} \backslash R\left(U_{F^{\text {gen }}}\right)$ has codimension at least 2 if $g+1$ is odd, and it is the Maroni divisor if $g+1$ is even (Proposition 3.1). The complement $\mathcal{H}_{4, g} \backslash U_{F}$ gen is the preimage $R^{-1}(Z)$. Therefore, the statements about the codimension follow from the finiteness of $R$.

For the question of reducibility, let $F=\mathcal{O}(k-1) \oplus \mathcal{O}(k+1)$ with $k=(g+3) / 2 \geq$ 3. The claim is that $C(F)$ is irreducible when $g>3$, and has two components when $g=3$. We have

$$
C(F)=\bigcup_{E} M(E, F)
$$

By Proposition 4.1, the varieties $M(E, F)$ are irreducible. Therefore, every component of $C(F)$ must be of the form $M(E, F)$ for some $E$.

Let $g>3$ and suppose $E \neq E^{\mathrm{gen}}$. The inclusion $M(E, F) \subset M(E)$ and Proposition 2.13 imply that $M(E, F)$ is a divisor if and only if $M(E, F)=M(E)$ and $E=\mathcal{O}(m-1) \oplus \mathcal{O}(m) \oplus \mathcal{O}(m+1)$. By choosing two generic quadrics as in (4-1) and (4-2), we can explicitly construct a curve in $M\left(E, F^{\text {gen }}\right)$, showing that $M(E, F) \neq M(E)$. Thus, it follows that the only component of $C(F)$ is $M\left(E^{\text {gen }}, F\right)$.

Example 4.8. The divisor $\mathcal{H}_{4, g} \backslash U_{F}$ gen is not irreducible for $g=3$. Indeed, take $F=\mathcal{O}(2) \oplus \mathcal{O}(4)$. Then $M\left(E^{\text {gen }}, F\right)$ is an irreducible component. Now consider the only other possibility for $E$, namely $E=\mathcal{O}(1) \oplus \mathcal{O}(2) \oplus \mathcal{O}(3)$. By (4-3), a cover in $M(E)$ must have $F=\mathcal{O}(2) \oplus \mathcal{O}(4)$. Furthermore, for this $E$ and $F$, we can choose the two quadrics generically and see that $M(E, F)$ is nonempty. Therefore, $M(E)=M(E, F)$ is another component of $\mathcal{H}_{4, g} \backslash U_{F}$ gen. 
Our next goal is to exhibit $U_{E^{\text {gen }}, F^{\text {gen }}}$ as a quotient. Let $\pi: \mathbb{P} E^{\text {gen }} \rightarrow \mathbb{P}^{1}$ be the projection. For brevity, set $E=E^{\text {gen }}$ and $F=F^{\text {gen }}$. Set

$$
V:=H^{0}\left(\mathbb{P}^{1}, F^{\vee} \otimes \operatorname{Sym}^{2} E\right) .
$$

An element $v \in \mathbb{P}_{\text {sub }} V$ corresponds to a map $\pi^{*} F(-2) \rightarrow \mathcal{O}_{\mathbb{P} E}$. Let $C_{v}$ be the zero locus of the image of this map. Let $V^{\circ} \subset \mathbb{P}_{\text {sub }} V$ be the open locus consisting of $v \in \mathbb{P}_{\text {sub }} V$ for which $C_{v}$ is irreducible and at worst nodal. Let $G_{F}:=\operatorname{Aut}\left(\mathbb{P} F / \mathbb{P}^{1}\right)$ and $G_{E}:=\operatorname{Aut}\left(\mathbb{P} E / \mathbb{P}^{1}\right)$. Then $G_{F} \times G_{E}$ acts on $V^{\circ}$. The assignment

$$
v \mapsto\left[\pi: C_{v} \rightarrow \mathbb{P}^{1}\right]
$$

defines a map

$$
q: V^{\circ} \rightarrow \tilde{\mathcal{H}}_{4, g}^{\dagger}
$$

Denote by $U_{E, F}^{\dagger}$ the preimage of $U_{E, F}$ under $\widetilde{\mathcal{H}}_{4, g}^{\dagger} \rightarrow \widetilde{\mathcal{H}}_{4, g}$.

Proposition 4.9. The image of $q$ is $U_{E^{\mathrm{gen}}, F \mathrm{gen}}^{\dagger}$. The fibers of $q$ consist of single G-orbits.

Proof. The proof is exactly analogous to the proof of Proposition 3.2.

Proposition 4.10 (Picard rank conjecture for degree 4). We have $\mathrm{Pic}_{\mathbb{Q}} \mathcal{H}_{4, g}=0$.

Proof. Retain the notation introduced above. For brevity, set $U=U_{E^{\text {gen }}, F^{\text {gen }}}$ and $U^{\dagger}=U_{E^{\text {gen }}, F^{\text {gen }}}^{\dagger}$ By Proposition 1.2 and Proposition 4.9, we have

$$
\begin{aligned}
\operatorname{rkPic} \operatorname{Pa}_{\mathbb{Q}} U \leq \operatorname{rkPic} U_{\mathbb{Q}} U^{\dagger}+\operatorname{rk} \chi\left(\operatorname{PGL}_{2}\right) & =\operatorname{rkPic} \operatorname{PiQ}_{\mathbb{Q}} U^{\dagger} \\
& \leq \operatorname{rk} \operatorname{Pic}_{\mathbb{Q}} V^{\circ}+\operatorname{rk} \chi(G) \leq 1+\operatorname{rk} \chi(G) .
\end{aligned}
$$

The final inequality follows because $V^{\circ}$ is an open subset of a projective space. Let $e$ be the number of divisorial components of $\widetilde{\mathcal{H}}_{3, g} \backslash U$. We then get the bound

$$
\operatorname{rkPic} \widetilde{\mathcal{H}}_{4, g} \leq \operatorname{rkPic}_{\mathbb{Q}} U+e \leq 1+\operatorname{rk} \chi(G)+e .
$$

Recall that $G=G_{F}$ gen $\times G_{E}$ gen .

If $g$ is an odd multiple of 3 , then

$$
G=\mathrm{PGL}_{2} \times \mathrm{PGL}_{3},
$$

$\operatorname{rk} \chi(G)=0$,

$e=2$ corresponding to $M$ in Proposition 4.5 and $C E$ in Proposition 4.7. 
If $g$ is odd, but not divisible by 3 , then

$$
\begin{aligned}
& G=\mathrm{PGL}_{2} \times G_{E} \\
& G_{E}=\left\{\left(\begin{array}{lll}
a & b & l_{1} \\
c & d & l_{2} \\
0 & 0 & e
\end{array}\right) \mid a, b, c, d, e \in \mathbb{C}, e(a d-b c) \in \mathbb{C}^{*}, l_{i} \in H^{0}\left(\mathbb{P}^{1}, \mathcal{O}(1)\right)\right\} / \mathbb{C}^{*}, \\
& \operatorname{rk} \chi(G)=1, \\
& e=1 \quad \text { corresponding to } C E \text { in Proposition 4.7. }
\end{aligned}
$$

If $g$ is even and divisible by 3 , then

$$
\begin{aligned}
G & =G_{F} \times \mathrm{PGL}_{2}, \\
G_{F} & =\left\{\left(\begin{array}{ll}
a & l \\
0 & b
\end{array}\right) \mid a, b \in \mathbb{C}^{*}, l \in H^{0}\left(\mathbb{P}^{1}, \mathcal{O}(1)\right)\right\} / \mathbb{C}^{*}, \\
\operatorname{rk} \chi(G) & =1, \\
e & =1 \quad \text { corresponding to } M \text { in Proposition } 4.5 .
\end{aligned}
$$

If $g$ is even and not divisible by 3 , then

$$
G=G_{F} \times G_{E} \text {, where } G_{F} \text { and } G_{E} \text { are as in the previous two cases, }
$$

$\operatorname{rk} \chi(G)=2$,

$$
e=0 \text {. }
$$

In all cases, we get

$$
\operatorname{rkPic} \widetilde{\mathcal{H}}_{4, g} \leq 3 .
$$

By Proposition 2.15, the classes in $\operatorname{Pic}_{\mathbb{Q}} \widetilde{\mathcal{H}}_{4, g}$ of the three components of $\widetilde{\mathcal{H}}_{4, g} \backslash \mathcal{H}_{4, g}$ are linearly independent. Therefore, we get $\mathrm{Pic}_{\mathfrak{Q}} \mathcal{H}_{4, g}=0$, as desired.

\section{Degree 5}

Let $C$ be a curve of genus $g$ and $\alpha: C \rightarrow \mathbb{P}^{1}$ a map of degree 5 . The relative canonical map embeds $C$ into a $\mathbb{P}^{3}$ bundle $\mathbb{P} E$ over $\mathbb{P}^{1}$, where $E$ is a vector bundle of rank 4 and degree $g+4$. The Casnati-Ekedahl structure theorem provides the following resolution of $\mathcal{O}_{C}$ :

$0 \longrightarrow \pi^{*} \operatorname{det} E(-5) \longrightarrow \pi^{*}\left(F^{\vee}(\operatorname{det} E)\right)(-3) \longrightarrow \pi^{*} F(-2) \longrightarrow \mathcal{O}_{\mathbb{P} E} \longrightarrow \mathcal{O}_{C} \longrightarrow 0$,

where $F$ is a vector bundle of rank 3 and degree $2 g+8$.

Explicitly, we can describe $C \subset \mathbb{P} E$ as follows. The resolution is determined completely by the middle map

$$
w: \pi^{*}\left(F^{\vee}(\operatorname{det} E)\right)(-3) \rightarrow \pi^{*} F(-2) .
$$


We can view this map as an element of the vector space $H^{0}\left(\mathbb{P}^{1}, F \otimes F \otimes E(-\operatorname{det} E)\right)$. Due to a theorem of [Casnati 1996], $w$ can be taken to be antisymmetric, that is, in the subspace

$$
V:=H^{0}\left(\mathbb{P}^{1}, \bigwedge^{2} F \otimes E \otimes \operatorname{det} E^{\vee}\right) .
$$

Even more explicitly, we can describe the defining equations of $C$ as follows. Let

$$
\begin{array}{ll}
F=\mathcal{O}\left(n_{1}\right) \oplus \cdots \oplus \mathcal{O}\left(n_{5}\right), & \text { where } n_{1} \leq \cdots \leq n_{5}, \text { and } \\
E=\mathcal{O}\left(m_{1}\right) \oplus \cdots \oplus \mathcal{O}\left(m_{4}\right), & \text { where } m_{1} \leq \cdots \leq m_{4} .
\end{array}
$$

We represent an element $w \in V$ by a skew symmetric matrix of forms

$$
M_{w}=\left(\begin{array}{ccccc}
0 & L_{1,2} & L_{1,3} & L_{1,4} & L_{1,5} \\
-L_{1,2} & 0 & L_{2,3} & L_{2,4} & L_{2,5} \\
-L_{1,3} & -L_{2,3} & 0 & L_{3,4} & L_{3,5} \\
-L_{1,4} & -L_{2,4} & -L_{3,4} & 0 & L_{4,5} \\
-L_{1,5} & -L_{2,5} & -L_{3,5} & -L_{4,5} & 0
\end{array}\right),
$$

where $L_{i, j} \in H^{0}\left(\mathbb{P}^{1}, E \otimes \operatorname{det} E^{\vee} \otimes \mathcal{O}\left(n_{i}+n_{j}\right)\right)$. In $\mathbb{P} E$, the curve $C_{w}$ is cut out by the $4 \times 4$ sub-Pfaffians of the matrix $M_{w}$.

The irreducibility of $C$ puts some restrictions on the possible matrices. Indeed, suppose

$$
L_{1,2}=L_{1,3}=0 \text {. }
$$

Then the Pfaffian $Q_{5}$ of the submatrix obtained by eliminating the fifth row and column is

$$
Q_{5}=L_{1,2} L_{3,4}-L_{1,3} L_{2,4}+L_{2,3} L_{1,4}=L_{2,3} L_{1,4} .
$$

Since $Q_{5}$ is reducible, $C_{w}$ is forced to be reducible.

Suppose further that $E=\mathcal{O}(k)^{r} \oplus \mathcal{O}(k+1)^{4-r}$, where $0 \leq r \leq 3$. Then the observation above implies that the maximum of the degrees of the summands of $E \otimes\left(\operatorname{det} E^{\vee}\right) \otimes \mathcal{O}\left(n_{1}+n_{3}\right)$ must be nonnegative, meaning

$$
n_{1}+n_{3}+k-(g+4) \geq-1 \text {. }
$$

Since the $n_{i}$ are increasing, we get the inequalities

$n_{i}+n_{j}+(k+1)-(g+4) \geq 0$

$$
\text { for every }(i, j) \text { with } i \neq j \text { except }(i, j)=(1,2) \text {. }
$$

Let $E^{\text {gen }}$ (resp. $F^{\text {gen }}$ ) be the most generic vector bundle on $\mathbb{P}^{1}$ of rank 4 (resp. 5) and degree $g+4$ (resp. $2 g+8$ ). Define $U_{E^{\text {gen }}}, U_{F}$ gen, and $U_{E^{\text {gen }}, F^{\text {gen }}}$ as before. These are the open subsets of $\tilde{\mathcal{H}}_{5, g}$ consisting of covers $\alpha$ for which $E_{\alpha}, F_{\alpha}$, and both $E_{\alpha}$ and $F_{\alpha}$ are the most generic. 
Proposition 5.1. The subvariety $M:=\widetilde{\mathcal{H}}_{5, g} \backslash U_{E^{\text {gen }}}$ has codimension at least 2 if $g$ is not divisible by 4 , and has a unique divisorial component if $g$ is divisible by 4 .

Proof. This is the degree-5 case of Proposition 2.13.

For the complement of $U_{F}$ gen, we must analyze the defining equations of $C$ in $\mathbb{P} E$.

Proposition 5.2. The subvariety $C E:=\mathcal{H}_{5, g} \backslash U_{F}$ gen has codimension at least 2 if $g+4$ is not a multiple of 5 (with the exception of $g=3$, in which case the complement parametrizes hyperelliptic curves), and contains a unique divisorial component if $g+4$ is a multiple of 5 .

Proof. We must characterize the Casnati-Ekedahl loci $C(F)$ which are divisorial. We have

$$
C(F)=\bigcup_{E} M(E, F)
$$

The loci $M(E, F)$ are irreducible by the same argument as in Proposition 4.1 - in the proof, just take $V=H^{0}\left(\mathbb{P}^{1}, \bigwedge^{2} F \otimes E \otimes \operatorname{det} E^{\vee}\right)$. Therefore, any component of $C(F)$ must be of the form $M(E, F)$. From the explicit description of degree-5 covers above, it is straightforward to compute that

$\operatorname{codim} M(E, F)=\operatorname{dim} \operatorname{Ext}^{1}(E, E)+\operatorname{dim} \operatorname{Ext}^{1}(F, F)-h^{1}\left(\bigwedge^{2} F \otimes E \otimes \operatorname{det} E^{\vee}\right)$.

Suppose $E \neq E^{\text {gen }}$. Then $M(E, F) \subset M(E)$. By Proposition 2.13, $M(E)$ has codimension at least 2 unless $E=\mathcal{O}(k) \oplus \mathcal{O}(k+1)^{\oplus d-3} \oplus \mathcal{O}(k+2)$. In this case, using the explicit description of degree- 5 covers, it is easy to construct covers $\alpha$ with $E_{\alpha}=E$ and $F_{\alpha}=F^{\text {gen }}$. Thus, $M(E, F) \neq M(E)$, and, since $M(E)$ is irreducible, $M(E, F) \subset M(E)$ has codimension at least 1 . Therefore, $M(E, F) \subset \mathcal{H}_{4, g}$ has codimension at least 2. Therefore, for $M(E, F)$ to be divisorial, we must have $E=E^{\mathrm{gen}}$. In this case, we have

$$
\operatorname{codim} M(E, F)=\operatorname{dim} \operatorname{Ext}^{1}(F, F)-h^{1}\left(\bigwedge^{2} F \otimes E \otimes \operatorname{det} E^{\vee}\right) .
$$

Suppose $h^{1}\left(\bigwedge^{2} F \otimes E \otimes \operatorname{det} E^{\vee}\right)=0$. Note that $\operatorname{dim} \operatorname{Ext}^{1}(F, F)=1$ if and only if

$$
F=\mathcal{O}(n-1) \oplus \mathcal{O}(n) \oplus \mathcal{O}(n) \oplus \mathcal{O}(n) \oplus \mathcal{O}(n+1) .
$$

In this case $5 n=2(g+4)$, and hence 5 divides $g+4$.

We are thus reduced to showing that $M(E, F)$ is not a divisor when $E=E^{\text {gen }}$ and

$$
h^{1}\left(\bigwedge^{2} F \otimes E(-\operatorname{det} E)\right)>0
$$


with the exception of $g=3$. Write

$$
E=\mathcal{O}(k)^{\oplus r} \oplus \mathcal{O}(k+1)^{\oplus 4-r}, \quad \text { where } 0 \leq r \leq 3,
$$

and

$$
F=\mathcal{O}\left(n_{1}\right) \oplus \mathcal{O}\left(n_{2}\right) \oplus \mathcal{O}\left(n_{3}\right) \oplus \mathcal{O}\left(n_{4}\right) \oplus \mathcal{O}\left(n_{5}\right), \quad \text { where } n_{1} \leq \cdots \leq n_{5}
$$

Consider an antisymmetric matrix

$$
M_{w}=\left(L_{i, j}\right), \quad 1 \leq i, j \leq 5,
$$

as in (5-1), representing an element of $H^{0}\left(\bigwedge^{2} F \otimes E \otimes \operatorname{det} E^{\vee}\right)$. Inequality (5-2) implies that any contribution to $h^{1}\left(\bigwedge^{2} F \otimes E \otimes \operatorname{det} E^{\vee}\right)$ must come from the $L_{1,2}$ entry. In other words, we have

$$
h^{1}\left(\bigwedge^{2} F \otimes E(-\operatorname{det} E)\right)=h^{1}\left(E \otimes \operatorname{det} E^{\vee} \otimes \mathcal{O}\left(n_{1}+n_{2}\right)\right) .
$$

Since $E=E^{\text {gen }}$, we have $h^{1}\left(E \otimes \operatorname{det} E^{\vee} \otimes \mathcal{O}\left(n_{1}+n_{2}\right)\right)>0$ if and only if

$$
n_{1}+n_{2}+(k+1)-(g+4)<0 \text {. }
$$

Hence, we get

$$
\begin{aligned}
h^{1}\left(E \otimes \operatorname{det} E^{\vee} \otimes \mathcal{O}\left(n_{1}+n_{2}\right)\right) & =4\left(-\left(n_{1}+n_{2}+k-(g+4)\right)-1\right)-(4-r) \\
& =4 g-4\left(n_{1}+n_{2}+k\right)+r+8 .
\end{aligned}
$$

Equation (5-2) tells us that $n_{1}+n_{3}+(k+1)-(g+4) \geq 0$, which implies $n_{2}<n_{3}$. Therefore,

$$
\operatorname{dim} \operatorname{Ext}^{1}(F, F) \geq\left(2 n_{5}+2 n_{4}+2 n_{3}\right)-3\left(n_{1}+n_{2}\right)-6 .
$$

Combining the two, we get

$\operatorname{dim} \operatorname{Ext}^{1}(F, F)-h^{1}\left(E \otimes \operatorname{det} E^{\vee} \otimes \mathcal{O}\left(n_{1}+n_{2}\right)\right)$

$$
\geq 2 n_{5}+2 n_{4}+2 n_{3}+n_{1}+n_{2}-3(g+4)-2 .
$$

Using $n_{1}+\cdots+n_{5}=2(g+4)$, the above inequality becomes

$$
\operatorname{dim} \operatorname{Ext}^{1}(F, F)-h^{1}\left(E \otimes \operatorname{det} E^{\vee} \otimes \mathcal{O}\left(n_{1}+n_{2}\right)\right) \geq(g+4)-\left(n_{1}+n_{2}\right)-2 .
$$

Finally, by using the assumption $n_{1}+n_{2}+(k+1)-(g+4)<0$, we conclude that $\operatorname{codim} M\left(E^{\mathrm{gen}}, F\right)=\operatorname{dim} \operatorname{Ext}^{1}(F, F)-h^{1}\left(E \otimes \operatorname{det} E^{\vee} \otimes \mathcal{O}\left(n_{1}+n_{2}\right)\right)>k-1$.

If $k>1$, then we get $\operatorname{codim} M\left(E^{\text {gen }}, F\right)>1$ as desired. We consider the cases where $k=1$ on an individual basis. These cases correspond to $0 \leq g \leq 4$. 
Case $g=4$. Then $E^{\text {gen }}=\mathcal{O}(2)^{\oplus 4}$ and $F^{\text {gen }}=\mathcal{O}(3)^{\oplus 4} \oplus \mathcal{O}(4)$. The relative canonical map embeds $C$ in $\mathbb{P} E^{\text {gen }} \simeq \mathbb{P}^{3} \times \mathbb{P}^{1}$. The projection to $\mathbb{P}^{3}$ restricts to the canonical map on $C$. Therefore, if $C$ is nonhyperelliptic, then there is only one quadric in $\mathbb{P}^{3}$ containing the canonical model of $C$. This means that the bundle $F$ has exactly one $\mathcal{O}(4)$ summand, and hence $F \cong F^{\text {gen }}$. The locus where $C$ is hyperelliptic is easily seen to be codimension- 2 in $\mathcal{H}_{5,4}$. This exhausts all possibilities in this case. Case $g=3$. Then $E^{\text {gen }}=\mathcal{O}(1) \oplus \mathcal{O}(2)^{\oplus 3}$ and $F^{\text {gen }}=\mathcal{O}(2) \oplus \mathcal{O}(3)^{\oplus 4}$. Consider the special bundle $F=\mathcal{O}(2) \oplus \mathcal{O}(2) \oplus \mathcal{O}(3)^{\oplus 2} \oplus \mathcal{O}(4)$. Then

$$
\operatorname{dim} \operatorname{Ext}^{1}(F, F)-h^{1}\left(\bigwedge^{2} F \otimes E \otimes \operatorname{det} E^{\vee}\right)=1 .
$$

Now consider a general $\left[\alpha: C \rightarrow \mathbb{P}^{1}\right] \in M(E, F) \subset \mathcal{H}_{5,3}$. Let $[X: Y: Z: W]$ denote the homogeneous coordinates (locally over $\mathbb{P}^{1}$ ) on $\mathbb{P} E$ corresponding to the summands of $E$. As usual, denote by $h$ the class of $\mathcal{O}_{\mathbb{P} E}(1)$ and by $f$ the class of the fiber of $\mathbb{P} E \rightarrow \mathbb{P}^{1}$. Since $\mathcal{O}(4)$ is a summand of $F$, there exists a unique effective divisor $Q$ of class $2 h-4 f$ on $\mathbb{P} E$ which contains $C$. The quadric $Q$ may be written as the zero locus of a form

$$
c_{0} Y^{2}+c_{1} Y Z+\cdots+c_{5} W^{2}
$$

where the $c_{i}$ are constants. Let $p: \mathbb{P} E \rightarrow \mathbb{P}^{2} \times \mathbb{P}^{1}$ be the projection from the section $[1: 0: 0: 0]$, and $g: \mathbb{P} E \rightarrow \mathbb{P}^{2} \times \mathbb{P}^{1} \rightarrow \mathbb{P}^{2}$ the composition with the projection onto the first factor. Then the rational map $g$ is given by the linear system $|h-2 f|$ on $\mathbb{P} E$, which restricts to the canonical series on $C$. However, the fact that $C$ lies on the relative quadric $Q$ means that the image $g(C)$ is exactly the conic defined by the equation for $Q$. Thus, $C$ is hyperelliptic.

Given the above geometric understanding of the $\mathcal{O}(4)$ summand of $F$, it is easy to show that if we begin with a hyperelliptic curve $C$, and a degree-5 map $\alpha: C \rightarrow \mathbb{P}^{1}$, then $F_{\alpha}$ must contain a unique $\mathcal{O}(4)$ summand. By the inequalities in (5-2), there are no other choices for $F$.

Case $g=1,2$. In these cases, we leave it to the reader to see that there are no nontrivial Casnati-Ekedahl or Maroni loci.

As before, we now exhibit $U_{E^{\text {gen }}, F^{\text {gen }}}$ as a quotient. For brevity, set $E=E^{\text {gen }}$ and $F=F^{\text {gen }}$. Set

$$
V:=H^{0}\left(\mathbb{P}^{1}, \bigwedge^{2} F \otimes E \otimes \operatorname{det} E\right) .
$$

An element $v \in \mathbb{P}_{\text {sub }} V$ defines an antisymmetric matrix as in (5-1). Let $C_{v}$ be the zero locus of the $4 \times 4$ sub-Pfaffians of this matrix. Let $V^{\circ} \subset \mathbb{P}_{\text {sub }} V$ be the open locus consisting of $v$ for which $C_{v}$ is irreducible and at worst nodal. Let $G_{F}:=\operatorname{Aut}\left(\mathbb{P} F / \mathbb{P}^{1}\right)$ and $G_{E}:=\operatorname{Aut}\left(\mathbb{P} E / \mathbb{P}^{1}\right)$. Then $G:=G_{F} \times G_{E}$ acts on $V^{\circ}$. 
The assignment $v \mapsto\left[\pi: C_{v} \rightarrow \mathbb{P}^{1}\right]$ defines a map

$$
q: V^{\circ} \rightarrow \tilde{\mathcal{H}}_{5, g}^{\dagger}
$$

Let $U_{E, F}^{\dagger}$ be the preimage of $U_{E^{\text {gen }}, F^{\text {gen }} \text { under }} \tilde{\mathcal{H}}_{5, g}^{\dagger} \rightarrow \widetilde{\mathcal{H}}_{5, g}$.

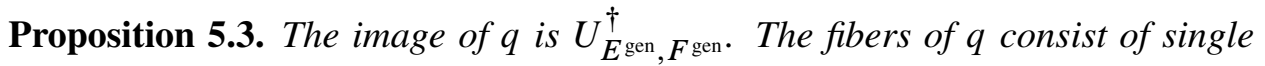
G-orbits.

Proof. The proof is exactly analogous to that of Proposition 3.2.

Proposition 5.4 (Picard rank conjecture for degree 5). We have $\operatorname{Pic}_{\mathbb{Q}} \mathcal{H}_{5, g}=0$.

Proof. The proof is entirely analogous to the proof of Proposition 4.10. We

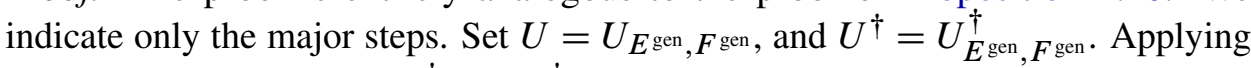
Proposition 1.2 to $V^{\circ} \rightarrow U^{\dagger}$ and $U^{\dagger} \rightarrow U$, we get

$$
\operatorname{rk} \operatorname{Pic}_{\mathbb{Q}} U \leq 1+\operatorname{rk} \chi(G) .
$$

Let $e$ be the number of divisorial components of $\tilde{\mathcal{H}}_{5, g} \backslash U$. We then get

$$
\operatorname{rkPic} \widetilde{\mathcal{H}}_{5, g} \leq 1+\operatorname{rk} \chi(G)+e .
$$

Both $G$ and $e$ depend on $g$ modulo 4 and 5. Using Propositions 5.1 and 5.2, we get the following possibilities:

\begin{tabular}{l|cl} 
& $\operatorname{rk} \chi(G)=\operatorname{rk} \chi\left(G_{E}\right)+\operatorname{rk} \chi\left(G_{F}\right)$ & \multicolumn{1}{c}{$e$} \\
\hline $4|g, 5| g+4$ & $0=0+0$ & $2(M$ and $C E)$ \\
$4 \mid g, 5 \nmid g+4$ & $1=0+1$ & $1(M)$ \\
$4 \nmid g, 5 \mid g+4$ & $1=1+0$ & $1(C E)$ \\
$4 \nmid g, 5 \nmid g+4$ & $2=1+1$ & 0
\end{tabular}

In all the cases, we have $\operatorname{Pic}_{\mathbb{Q}} \widetilde{\mathcal{H}}_{5, g} \leq 3$. Combined with Proposition 2.15, this gives $\operatorname{Pic}_{\mathbb{Q}} \mathcal{H}_{5, g}=0$.

\section{From Hurwitz spaces to Severi varieties}

The associated scroll construction in Section 2A lets us relate the Picard rank of a Hurwitz space to the Picard rank of a Severi variety. In this section, we work out this relation.

Recall the notation $\mathcal{U}_{g}\left(\mathbb{F}_{m}, d \tau\right), \mathcal{V}_{g}\left(\mathbb{F}_{m}, d \tau\right)$, and $\mathcal{V}_{g}^{\text {irr }}\left(\mathbb{F}_{m}, d \tau\right)$ from page 462. When confusion is unlikely, we abbreviate them by $\mathcal{U}, \mathcal{V}$, and $\mathcal{V}^{\text {irr }}$. Following [Diaz and Harris 1988a], we enlarge $\mathcal{U}$ by including the irreducible curves of geometric genus $g$ having a cusp, a tacnode, a triple point, and irreducible nodal curves of geometric genus $(g-1)$ (that is, curves having an "additional" node). Note that the resulting enlargement of $\mathcal{U}$ is a partial compactification of $\mathcal{U}$ in the linear system 
$|d \tau|$. Although it does not include extremely singular degenerations of nodal curves, it does include all codimension-1 degenerations (Proposition 6.3). Denote by $\tilde{\mathcal{U}}$ the normalization of this partial compactification. The local analysis from [Diaz and Harris 1988a, §1] of the Severi variety at points corresponding to cusps, tacnodes, triple points, and an additional node shows that $\tilde{\mathcal{U}}$ is smooth. Since $\tilde{\mathcal{U}}$ maps to the linear series $|d \tau|$, it carries over it a family of (singular) curves. The normalization of the total space of this family gives a family $\mathcal{C} \rightarrow \widetilde{\mathcal{U}}$ of curves of arithmetic genus $g$. A generic fiber of $\mathcal{C} \rightarrow \tilde{\mathcal{U}}$ is the normalization the corresponding curve on $\mathbb{F}_{m}$.

Using the universal family, we can construct tautological divisor classes on $\tilde{\mathcal{U}}$ as follows. Consider the diagram

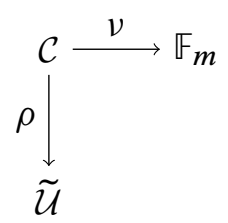

Define five tautological divisor classes on $\tilde{\mathcal{U}}$ (The subscript $s$ stands for "Severi"):

(1) $\lambda_{s}:=c_{1}\left(\rho_{*} \omega_{\rho}\right)$.

(2) $\kappa_{s}:=\rho_{*}\left(c_{1}\left(\omega_{\rho}\right)^{2}\right)$.

(3) $\xi_{s}:=\rho_{*}\left(v^{*}(f) \cdot c_{1}\left(\omega_{\rho}\right)\right)$.

(4) $\theta_{s}:=\rho_{*}\left(v^{*}(\sigma) \cdot c_{1}\left(\omega_{\rho}\right)\right)$.

(5) $\psi_{s}:=\rho_{*}\left(v^{*}[\right.$ point $\left.]\right)$.

Since the irreducible curves in the linear system $|d \tau|$ avoid the directrix $\sigma$, we get $\theta_{s}=\psi_{s}=0$. Therefore, it is natural to conjecture:

Conjecture 6.1. The rational Picard group of $\tilde{\mathcal{U}}$ is tautological, that is,

$$
\operatorname{Pic}_{\mathbb{Q}} \tilde{\mathcal{U}}=\mathbb{Q}\left\langle\lambda_{s}, \kappa_{s}, \xi_{s}\right\rangle .
$$

Denote by CU, TN, TP, and $\Delta$ the closures in $\mathcal{V}^{\text {irr }}$ of the locus curves with a cusp, tacnode, triple point, or an additional node, respectively. Abusing notation, denote their preimages in $\tilde{\mathcal{U}}$ by the same letters.

Remark 6.2. It is not hard to check that the classes in $\operatorname{Pic}_{\mathbb{Q}} \tilde{\mathcal{U}}$ of $\mathrm{CU}, \mathrm{TN}$, TP, and $\Delta$ can be expressed as $\mathbb{Q}$-linear combinations of $\lambda_{s}, \kappa_{s}$, and $\xi_{s}$ and vice versa. Conjecture 6.1 is therefore equivalent to

$$
\operatorname{Pic}_{\mathbb{Q}} \mathcal{U}=0 .
$$

Proposition 6.3. The only divisorial components of $\mathcal{V}^{\mathrm{irr}} \backslash \mathcal{U}$ are $\mathrm{CU}, \mathrm{TN}, \mathrm{TP}$, and $\Delta$. 
Proof. It suffices to show that the codimension-1 components of $\mathcal{V} \backslash \mathcal{U}$ are the loci of curves with cusps, tacnodes, triple points or an additional node. This follows by the same proof as for Theorem 1.4 in [Diaz and Harris 1988b]. The critical ingredient of the argument is provided by Lemma 6.4 .

Lemma 6.4. Let $D \in|d \tau|$ be a reduced irreducible curve on the Hirzebruch surface $\mathbb{F}_{m}$. Denote by $A$ the conductor ideal of the singularities of $D$. Then A imposes independent conditions on $H^{0}\left(\mathbb{F}_{m}, \mathcal{O}(d \tau)\right)$.

Proof. Let $K=K_{\mathbb{F}_{m}}$ be the canonical class. The anticanonical class $-K$ is effective. Furthermore, the fixed component of $-K$ is the directrix $\sigma$, and $-K$ separates points away from $\sigma$. It is classical that $A$ imposes independent conditions on the adjoint linear system $|K+D|$. Let $Z=V(A)$ be the zero-dimensional scheme defined by the ideal sheaf $A$. Then the restriction map

$$
H^{0}(\mathcal{O}(K+D)) \rightarrow H^{0}\left(\mathcal{O}_{Z}(K+D)\right)
$$

is surjective. Therefore, we can conclude the same for

$$
H^{0}(\mathcal{O}(D)) \rightarrow H^{0}\left(\mathcal{O}_{Z}(D)\right)
$$

by multiplying the previous restriction map by a general section of $\mathcal{O}(-K)$.

We now rephrase the Picard rank conjecture for Hurwitz spaces in a manner similar to Conjecture 6.1. Consider the diagram

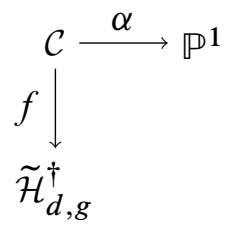

Define the following tautological divisor classes on $\widetilde{\mathcal{H}}_{d, g}^{\dagger}$ (The subscript " $\mathrm{h}$ " stands for "Hurwitz"):

(1) $\lambda_{h}:=c_{1}\left(f_{*} \omega_{f}\right)$.

(2) $\kappa_{h}:=f_{*}\left(c_{1}\left(\omega_{f}\right)^{2}\right)$.

(3) $\xi_{h}:=f_{*}\left(\alpha^{*}[\right.$ point $\left.] \cdot c_{1}\left(\omega_{f}\right)\right)$.

Conjecture 6.5. The rational Picard group of $\tilde{\mathcal{H}}_{d, g}^{\dagger}$ is tautological, that is,

$$
\operatorname{Pic}_{\mathbb{Q}} \tilde{\mathcal{H}}_{d, g}^{\dagger}=\mathbb{Q}\left\langle\lambda_{h}, \kappa_{h}, \xi_{h}\right\rangle
$$


Remark 6.6. It is easy to see that the classes of $T, D$, and $\Delta$ can be expressed as $\mathbb{Q}$ linear combinations of $\lambda_{h}, \kappa_{h}$, and $\xi_{h}$ and vice versa. Also, by Proposition 1.3, the framed/unframed distinction is irrelevant. Therefore, Conjecture 6.5 is equivalent to the Picard rank conjecture stated in the introduction, namely, that

$$
\operatorname{Pic}_{\mathbb{Q}} \mathcal{H}_{d, g}=0
$$

We now state the main theorem of this section:

Theorem 6.7. If $m \geq\lfloor(g+d-1) /(d-1)\rfloor$, then Conjecture 6.1 for $\tilde{\mathcal{U}}_{g}\left(\mathbb{F}_{m}, d \tau\right)$ implies Conjecture 6.5 for $\tilde{\mathcal{H}}_{d, g}^{\dagger}$. If $m \geq\lceil 2(g+d-1) / d\rceil$, then Conjecture 6.1 for $\tilde{\mathcal{U}}_{g}\left(\mathbb{F}_{m}, d \tau\right)$ is equivalent to Conjecture 6.5 for $\tilde{\mathcal{H}}_{d, g}^{\dagger}$.

Proof. Let $m \geq\lfloor(g+d-1) /(d-1)\rfloor$. Retain the notation introduced in this section. In particular, abbreviate $\mathcal{U}_{g}\left(\mathbb{F}_{m}, d \tau\right)$ by $\mathcal{U}$, and so on. Let $\pi: \mathbb{F}_{m} \rightarrow \mathbb{P}^{1}$ be the projection and $\sigma \subset \mathbb{F}_{m}$ the directrix. Fix a section $\zeta \in H^{0}\left(\mathbb{F}_{m}, \pi^{*} \mathcal{O}(m)\right)$ corresponding to a smooth element of the linear series $|\tau|$. We view $\mathbb{F}_{m} \backslash \sigma$ as the total space of the line bundle $\mathcal{O}(m)$ on $\mathbb{P}^{1}$ and $\zeta$ as the tautological section of $\pi^{*} \mathcal{O}(m)$ on this total space.

Let $\phi: \mathcal{C} \rightarrow \mathbb{P}^{1}$ be the composition $\phi=\pi \circ v$. Let $Z \subset \tilde{\mathcal{U}}$ be the open subset consisting of the $u$ where $h^{0}\left(\mathcal{C}_{u}, \phi^{*} \mathcal{O}(m)\right)$ is minimal. Likewise, let $W \subset \widetilde{\mathcal{H}}_{d, g}^{\dagger}$ be the subset consisting of $\left[\alpha: C \rightarrow \mathbb{P}^{1}\right]$ where $h^{0}\left(C, \alpha^{*} \mathcal{O}(m)\right)$ is minimal. By Proposition 2.13, the complement of $W$ in $\widetilde{\mathcal{H}}_{d, g}^{\dagger}$ has codimension at least 2. Let $V$ be the total space of the vector bundle $\left.f_{*} \alpha^{*} \mathcal{O}(m)\right|_{W}$ over $W$.

We have a birational morphism $q: Z \rightarrow V$ defined as follows. A point $u \in \tilde{\mathcal{U}}$ is mapped to $\left[\phi_{u}: \mathcal{C}_{u} \rightarrow \mathbb{P}^{1}, v\right]$, where $v \in H^{0}\left(\mathcal{C}_{u}, \phi_{u}^{*} \mathcal{O}(m)\right)$ is the restriction of $\zeta$. To define the inverse, we must restrict to an open subset of $V$. Let $X \subset V$ be the open subset consisting of $\left(\left[\alpha: C \rightarrow \mathbb{P}^{1}\right], v\right)$, where $v \in H^{0}\left(C, \alpha^{*} \mathcal{O}(m)\right)$ is such that the lift of $C \rightarrow \mathbb{P}^{1}$ to $C \rightarrow \mathbb{F}_{m}$ defined by $v$ is birational onto its image. We then get a morphism $p: X \rightarrow \mathcal{V}^{\text {irr }}$ which is quasifinite and generically one-to-one. Let $Y \subset X$ be the open subset consisting of points whose associated element in $\mathcal{V}^{\text {irr }}$ has at worst a cusp, a tacnode, a triple point, or an additional node. By Proposition 6.3 and the quasifiniteness of $p$, the complement of $Y$ in $X$ has codimension at least 2. Since $Y$ is normal, we get a morphism $p: Y \rightarrow Z \subset \tilde{\mathcal{U}}$, inverse to $q$. We summarize the spaces we have defined and their relationships in the following diagram:

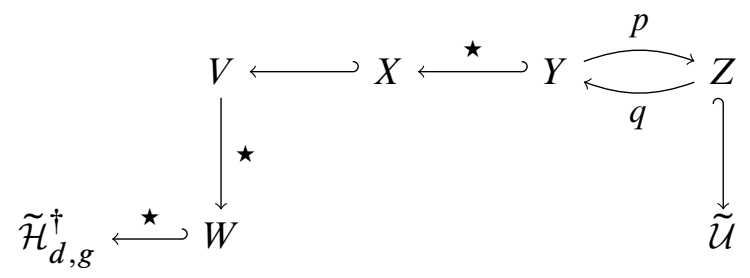


The inclusions are open inclusions. $Y$ and $Z$ are isomorphic via $p$ and $q$. The maps marked by $\star$ induce isomorphisms on Picard groups. For the open inclusions, this is because the complements have codimension at least 2. For $V \rightarrow W$, this is because it is a vector bundle.

Denote the pullbacks of $\lambda_{h}, \kappa_{h}$, and $\xi_{h}$ to $W, V, X$, and $Y$ by the same letters. Then, we have

$$
\begin{array}{lll}
p^{*} \lambda_{s}=\lambda_{h}, & p^{*} \kappa_{s}=\kappa_{h}, & p^{*} \xi_{s}=\xi_{h}, \\
q^{*} \lambda_{h}=\lambda_{s}, & q^{*} \kappa_{h}=\kappa_{s}, & q^{*} \xi_{h}=\xi_{s} .
\end{array}
$$

We may thus drop the subscripts and use $\lambda, \kappa$, and $\xi$ to denote the corresponding divisors on any of the spaces in (6-1).

Before we proceed, we must comment on the inclusion $X \hookrightarrow V$. The complement consists of $\left(\left[\alpha: C \rightarrow \mathbb{P}^{1}\right], v\right)$, where $v \in H^{0}\left(C, \alpha^{*} \mathcal{O}(m)\right)$ does not give a birational map to $\mathbb{F}_{m}$. Let us disregard the $\alpha$ that factor nontrivially (such $\alpha$ form a set of codimension at least 2). Then the only such $v$ are the pullbacks of the sections in $H^{0}\left(\mathbb{P}^{1}, \mathcal{O}(m)\right)$. The locus $\left(\left[\alpha: C \rightarrow \mathbb{P}^{1}\right], v\right)$ where $v \in \alpha^{*} H^{0}\left(\mathbb{P}^{1}, \mathcal{O}(m)\right)$ has codimension at least 2 except in the case $g \equiv-1(\bmod (d-1))$ and $m=$ $\lfloor(g+d-1) /(d-1)\rfloor$, that is, when the generic splitting of $\alpha_{*} \mathcal{O}_{C}$ is

$$
\alpha_{*} \mathcal{O}_{C}=\mathcal{O} \oplus \mathcal{O}(-m) \oplus \mathcal{O}(-m-1) \oplus \cdots \oplus \mathcal{O}(-m-1)
$$

In this case, the complement of $X$ in $V$ has a divisorial component given by the image of the constant vector bundle $H^{0}\left(\mathbb{P}^{1}, \mathcal{O}(m)\right) \otimes \mathcal{O}_{W}$. However, the class of this divisor in $\operatorname{Pic}_{\mathbb{Q}} V \cong \operatorname{Pic}_{\mathbb{Q}} W$ is in the span of $\lambda, \kappa$, and $\xi$. Therefore, in any case, $\operatorname{Pic}_{\mathbb{Q}} V$ is spanned by $\lambda, \kappa$, and $\xi$ if and only if $\operatorname{Pic}_{\mathbb{Q}} X$ is.

Assume that Conjecture 6.1 holds. From diagram (6-1), we see that $\operatorname{Pic}_{\mathbb{Q}} X$ is spanned by $\lambda, \kappa$, and $\xi$. By the comment about $X \hookrightarrow V$ above, this implies that $\operatorname{Pic}_{\mathbb{Q}} V$, and in turn $\operatorname{Pic}_{\mathbb{Q}} \tilde{\mathcal{H}}_{d, g}^{\dagger}$, is spanned by $\lambda, \kappa$, and $\xi$. Hence Conjecture 6.5 holds.

Assume that $m \geq\lceil 2(g+d-1) /(d-1)\rceil$ and Conjecture 6.5 holds. Then, by Proposition 2.6 the inclusion $Z \hookrightarrow \widetilde{\mathcal{U}}$ is in fact an isomorphism. Again, diagram (6-1) shows that $\operatorname{Pic}_{\mathbb{Q}} \tilde{\mathcal{U}}$ is spanned by $\lambda$, $\kappa$, and $\xi$. Hence Conjecture 6.1 holds.

\section{Acknowledgments}

We are grateful to our advisor Joe Harris for suggesting this problem and for providing constant support and encouragement. We also thank Gabriel Bujokas, Dawei Chen, Maksym Fedorchuk, and Ravi Vakil for helpful comments and conversations. 


\section{References}

[Bolognesi and Lönne 2014] M. Bolognesi and M. Lönne, "Mapping Class Groups of Trigonal Loci”, preprint, 2014. arXiv 1403.7399

[Bolognesi and Vistoli 2012] M. Bolognesi and A. Vistoli, "Stacks of trigonal curves", Trans. Amer. Math. Soc. 364:7 (2012), 3365-3393. MR 2901217 Zbl 06188646

[Casnati 1996] G. Casnati, "Covers of algebraic varieties, II: Covers of degree 5 and construction of surfaces”, J. Algebraic Geom. 5:3 (1996), 461-477. MR 97c:14015 Zbl 0921.14006

[Casnati 1998] G. Casnati, "Covers of algebraic varieties, III: The discriminant of a cover of degree 4 and the trigonal construction", Trans. Amer. Math. Soc. 350:4 (1998), 1359-1378. MR 98i:14021

[Casnati and Ekedahl 1996] G. Casnati and T. Ekedahl, "Covers of algebraic varieties, I: A general structure theorem, covers of degree 3, 4 and Enriques surfaces”, J. Algebraic Geom. 5:3 (1996), 439-460. MR 97c:14014 Zbl 0866.14009

[Coppens 1999] M. Coppens, "Existence of pencils with prescribed scrollar invariants of some general type”, Osaka J. Math. 36:4 (1999), 1049-1057. MR 2001g:14006 Zbl 1047.14500

[Cornalba 2007] M. Cornalba, "The Picard group of the moduli stack of stable hyperelliptic curves", Atti Accad. Naz. Lincei Cl. Sci. Fis. Mat. Natur. Rend. Lincei (9) Mat. Appl. 18:1 (2007), 109-115. MR 2008b:14017 Zbl 1222.14054

[Cornalba and Harris 1988] M. Cornalba and J. Harris, "Divisor classes associated to families of stable varieties, with applications to the moduli space of curves", Ann. Sci. École Norm. Sup. (4) 21:3 (1988), 455-475. MR 89j:14019 Zbl 0674.14006

[Diaz and Edidin 1996] S. Diaz and D. Edidin, "Towards the homology of Hurwitz spaces", $J$. Differential Geom. 43:1 (1996), 66-98. MR 98e:14028 Zbl 0848.14005

[Diaz and Harris 1988a] S. Diaz and J. Harris, "Geometry of the Severi variety", Trans. Amer. Math. Soc. 309:1 (1988), 1-34. MR 89i:14018 Zbl 0677.14003

[Diaz and Harris 1988b] S. Diaz and J. Harris, "Ideals associated to deformations of singular plane curves”, Trans. Amer. Math. Soc. 309:2 (1988), 433-468. MR 89m:14003 Zbl 0707.14022

[Donagi 1981] R. Donagi, "The tetragonal construction", Bull. Amer. Math. Soc. (N.S.) 4:2 (1981), 181-185. MR 82a:14009 Zbl 0491.14016

[Harer 1983] J. Harer, "The second homology group of the mapping class group of an orientable surface”, Invent. Math. 72:2 (1983), 221-239. MR 84g:57006 Zbl 0533.57003

[Harris 1986] J. Harris, “On the Severi problem”, Invent. Math. 84:3 (1986), 445-461. MR 87f:14012 Zbl 0596.14017

[Knop et al. 1989] F. Knop, H. Kraft, and T. Vust, “The Picard group of a $G$-variety”, pp. 77-87 in Algebraische Transformationsgruppen und Invariantentheorie, edited by H. Kraft et al., DMV Sem. 13, Birkhäuser, Basel, 1989. MR 1044586 Zbl 0705.14005

[Mochizuki 1995] S. Mochizuki, "The geometry of the compactification of the Hurwitz scheme", Publ. Res. Inst. Math. Sci. 31:3 (1995), 355-441. MR 96j:14017 Zbl 0866.14013

[Mumford et al. 1994] D. Mumford, J. Fogarty, and F. Kirwan, Geometric invariant theory, 3rd ed., Ergebnisse der Mathematik und ihrer Grenzgebiete (2) [Results in Mathematics and Related Areas (2)] 34, Springer, Berlin, 1994. MR 95m:14012 Zbl 0797.14004

[Ohbuchi 1997] A. Ohbuchi, "On some numerical relations of $d$-gonal linear systems", J. Math. Tokushima Univ. 31 (1997), 7-10. MR 99b:14005 Zbl 0938.14011

[Recillas 1973] S. Recillas, "Maps between Hurwitz spaces", Bol. Soc. Mat. Mexicana (2) 18 (1973), 59-63. MR 50 \#13050 Zbl 0313.14003 
[Stankova-Frenkel 2000] Z. E. Stankova-Frenkel, "Moduli of trigonal curves", J. Algebraic Geom. 9:4 (2000), 607-662. MR 2001h:14031 Zbl 1001.14007

[Teissier 1980] B. Teissier, "Résolution simultanée, I: Familles de courbes", pp. viii+339 in Séminaire sur les singularités des surfaces (Palaiseau, 1976-1977), edited by M. Demazure et al., Lecture Notes in Mathematics 777, Springer, Berlin, 1980. MR 82d:14021 Zbl 0464.14005

[Tyomkin 2007] I. Tyomkin, "On Severi varieties on Hirzebruch surfaces", Int. Math. Res. Not. 2007:23 (2007), 31. MR 2008m:14048 Zbl 1135.14026

Communicated by Ravi Vakil

Received 2014-07-27 Revised 2014-11-04 Accepted 2014-12-11

anandrd@math.columbia.edu Department of Mathematics, Columbia University, 2990 Broadway, New York, NY 10027, United States

anand.patel@bc.edu

Mathematics Department, Boston College, Carney Hall, Chestnut Hill, MA 02467, United States 


\section{Algebra \& Number Theory}

msp.org/ant

\section{EDITORS}

MANAGING EDITOR

Bjorn Poonen

Massachusetts Institute of Technology

Cambridge, USA

\author{
EDITORIAL BOARD CHAIR \\ David Eisenbud \\ University of California \\ Berkeley, USA
}

BOARD OF EDITORS

Georgia Benkart

Dave Benson

Richard E. Borcherds

John H. Coates

J-L. Colliot-Thélène

Brian D. Conrad

Hélène Esnault

Hubert Flenner

Edward Frenkel

Andrew Granville

Joseph Gubeladze

Roger Heath-Brown

Craig Huneke

János Kollár

Yuri Manin

Barry Mazur

Philippe Michel

Susan Montgomery
University of Wisconsin, Madison, USA

University of Aberdeen, Scotland

University of California, Berkeley, USA

University of Cambridge, UK

CNRS, Université Paris-Sud, France

University of Michigan, USA

Freie Universität Berlin, Germany

Ruhr-Universität, Germany

University of California, Berkeley, USA

Université de Montréal, Canada

San Francisco State University, USA

Oxford University, UK

University of Virginia, USA

Princeton University, USA

Northwestern University, USA

Harvard University, USA

École Polytechnique Fédérale de Lausanne

University of Southern California, USA
Shigefumi Mori

Raman Parimala

Jonathan Pila

Anand Pillay

Victor Reiner

Peter Sarnak

Joseph H. Silverman

Michael Singer

Vasudevan Srinivas

J. Toby Stafford

Richard Taylor

Ravi Vakil

Michel van den Bergh

Marie-France Vignéras

Kei-Ichi Watanabe

Efim Zelmanov

Shou-Wu Zhang
RIMS, Kyoto University, Japan

Emory University, USA

University of Oxford, UK

University of Notre Dame, USA

University of Minnesota, USA

Princeton University, USA

Brown University, USA

North Carolina State University, USA

Tata Inst. of Fund. Research, India

University of Michigan, USA

Harvard University, USA

Stanford University, USA

Hasselt University, Belgium

Université Paris VII, France

Nihon University, Japan

University of California, San Diego, USA

Princeton University, USA

\section{PRODUCTION}

production@msp.org

Silvio Levy, Scientific Editor

See inside back cover or msp.org/ant for submission instructions.

The subscription price for 2015 is US \$255/year for the electronic version, and $\$ 440 /$ year ( $\$ 55$, if shipping outside the US) for print and electronic. Subscriptions, requests for back issues and changes of subscribers address should be sent to MSP.

Algebra \& Number Theory (ISSN 1944-7833 electronic, 1937-0652 printed) at Mathematical Sciences Publishers, 798 Evans Hall \#3840, c/o University of California, Berkeley, CA 94720-3840 is published continuously online. Periodical rate postage paid at Berkeley, CA 94704, and additional mailing offices.

ANT peer review and production are managed by EditFLOW ${ }^{\circledR}$ from MSP.

\section{PUBLISHED BY}

7 mathematical sciences publishers

nonprofit scientific publishing

http://msp.org/

(C) 2015 Mathematical Sciences Publishers 


\section{Algebra \& Number Theory}

Volume $9 \quad$ No. $2 \quad 2015$

Lifting harmonic morphisms II: Tropical curves and metrized complexes

OMID AMINI, MATTHEW BAKER, ERWAN BRUgallé and JosePH RABINOFF

Noncommutative Hilbert modular symbols

IVAN HOROZOV

$p$-adic Hodge theory in rigid analytic families

REBECCA BELLOVIN

Semistable periods of finite slope families

RUOCHUAN LIU

ANAND DEOPURKAR and ANAND PATEL

Finite-dimensional quotients of Hecke algebras

493

IVAN LOSEV

Semiample invertible sheaves with semipositive continuous hermitian metrics

503 ATSUSHI MORIWAKI 